\title{
Necessary Conditions for Well-Posedness of the Flat Cauchy Problem and the Regularity-Loss of Solutions
}

\author{
By \\ Takeshi MANDAI*
}

\section{§0. Introduction}

It is well-known that for some weakly hyperbolic operators, the regularityloss of solutions depends largely on lower order terms. For example, if $P$ has the principal part $\partial_{t}^{2}-t^{2 k} \partial_{x}^{2}$ and the Cauchy problem for $P$ with the initial surface $t=0$ is well-posed, then the lower order terms have the form $a \partial_{t}+$ $t^{k-1} b \partial_{x}+c$ where $a, b, c$ are $C^{\infty}$-functions. And the regularity-loss depends on $|\operatorname{Re} b(0, x)|$. In this paper, we pay attention to the behavior of the principal part with respect to the time variable $t$, and we give a result of the following type (cf. [2], [3] and their references).

Assume that the (flat) Cauchy problem is well-posed. Then, the lower order terms satisfy some conditions, and the regularity-loss of solutions depends on certain quantities, which are determined by lower order terms.

We observe another example. For the operator $P=\partial_{t}^{2}-t \partial_{x}^{2}+a \partial_{t}+b \partial_{x}+c$ where $a, b, c$ are $C^{\infty}$-functions, the regularity-loss of solutions does not depend on lower order terms. But, if we consider the operator $P=\partial_{t}^{2}-t \partial_{x}^{2}+a \partial_{t}+t^{-1 / 2} b \partial_{x}$ $+c$, then the regularity-loss depends on $|\operatorname{Re} b(0, x)|$. We want also to deal Fuchsian operators. So, we consider the operators whose coefficients may have fractional or negative powers of $t$. For these operators, we can consider the flat Cauchy problem to which the non-characteristic Cauchy problem for operators with $C^{\infty}$-coefficients can be easily reduced.

Our program is as follows. In Section 1, we state definitions, the result and some examples. Our result consists of three theorems. In Section 2, we consider two transformations of operators which reduce the theorems to easier situation. In Section 3, we study an elementary fact on Newton polygons and apply it. In Sections 4, 5 and 6, we prove the theorems.

Communicated by S. Matsuura, February 13, 1982.

* Research Institute for Mathematical Sciences, Kyoto University, Kyoto 606, Japan. 


\section{$\S 1$. The Result and Examples}

First we introduce some notations.

$$
\begin{aligned}
& (t, x)=\left(t, x^{\prime}, x_{n}\right)=\left(t, x_{1}, \cdots, x_{n-1}, x_{n}\right) \in \boldsymbol{R}^{n+1}, \\
& (\tau, \xi)=\left(\tau, \xi^{\prime}, \xi_{n}\right)=\left(\tau, \xi_{1}, \cdots, \xi_{n-1}, \xi_{n}\right) \in \boldsymbol{R}^{n+1} . \\
& \partial_{t}=\partial / \partial t, \partial_{x_{j}}=\partial / \partial x_{j}, D_{t}=-i \partial_{t}, D_{x_{j}}=-i \partial_{x_{j}}, \\
& D_{x}^{\alpha}=D_{x_{1}}^{\alpha_{1}} \cdots D_{x_{n}}^{\alpha},
\end{aligned}
$$

where $\alpha=\left(\alpha_{1}, \cdots, \alpha_{n}\right)$ is a multi-index. For an open set $\Omega$ in $\mathbb{R}^{n+1}$, put

$$
\begin{aligned}
& \Omega^{+}=\{(t, x) \in \Omega ; t \geqq 0\}, \quad \Omega_{\theta}^{+}=\{(t, x) \in \Omega ; 0 \leqq t \leqq \theta\} . \\
& E_{M}\left(\Omega^{+}\right)=\left\{f(t, x) ; f\left(t^{M}, x\right) \in C^{\infty}\left(\Omega^{+}\right)\right\}
\end{aligned}
$$

where $M$ is a positive integer, and

$$
\begin{aligned}
E\left(\Omega^{+}\right) & =\bigcup_{M>0} E_{M}\left(\Omega^{+}\right) . \\
C_{f}^{\infty}\left(\Omega^{+}\right) & =\left\{g \in C^{\infty}\left(\Omega^{+}\right) ; g \text { is flat at } t=0\right\} \\
& =\left\{g \in C^{\infty}(\Omega) ; g(t, x)=0 \text { if } t \leqq 0\right\} .
\end{aligned}
$$

Let $P$ be a differential operator on $[0, T] \times \boldsymbol{R}^{n}(T>0)$ and

$$
P=\sum_{j+|\alpha| \leqq m} a_{j, \alpha}(t, x) D_{t}^{j} D_{x}^{\alpha}=\sum_{h=0}^{m} P_{m-h}\left(t, x ; D_{t}, D_{x}\right),
$$

where $P_{m-h}$ is the homogeneous part of degree $m-h$.

For a positive integer $M$ and a positive rational number $\mu_{0}$ where $\mu_{0} M$ is an integer, we consider the following condition:

$$
\begin{array}{ll}
\left(A-M, \mu_{0}\right) & a_{j, \alpha} \in t^{j+\mu_{0} \mid \alpha_{i}-m} \times E_{M}\left([0, T] \times \boldsymbol{R}^{n}\right) \\
& a_{m, 0}(t, x) \equiv 1 \text { on }[0, T] \times \boldsymbol{R}^{n} .
\end{array}
$$

In other words,

$$
\begin{array}{ll}
\left(A-M, \mu_{0}\right)^{\prime} \quad t^{m} P(t, x ; \tau, \xi)=\sum_{j+|\alpha| \leqslant m} a_{j, \alpha}^{\prime}(t, x)(t \tau)^{j}\left(t^{\mu_{0}} \xi\right)^{\alpha}, \\
& \text { where } \quad a_{j, \alpha}^{\prime} \in E_{M}\left([0, T] \times \boldsymbol{R}^{n}\right), \quad a_{m, 0}^{\prime}(t, x) \equiv 1 .
\end{array}
$$

Thus, we consider the operators of Fuchsian type with $C^{\infty}$-coefficients with respect to $\left(t^{1 / M}, x\right)$.

For these operators, we can consider the flat Cauchy problem; for $f \in C_{f}^{\infty}\left(\Omega^{+}\right)$, seek the solution $u \in C_{f}^{\infty}\left(\Omega^{+}\right)$of $P u=f$.

Definition 1.1. We say that the flat Cauchy problem for $P$ is $\left(\mu_{0}\right)$-well-posed at the origin in $\boldsymbol{R}^{n+1}$, if there exist an open neighborhood $\Omega$ of the origin and a positive constant $C_{0}$ such that the following two conditions are satisfied.

(E) For any $f \in C_{f}^{\infty}\left(\Omega^{+}\right)$, there exists $u \in C_{f}^{\infty}\left(\Omega^{+}\right)$such that $P u=f$ in $\Omega^{+}$.

(U) $\left\{\begin{array}{l}\text { For any }(\hat{t}, \hat{x}) \in \Omega^{+} \text {which satisfies } \Gamma=\Gamma_{\mu_{0}}\left(\hat{t}, \hat{x} ; C_{0}\right) \subset \Omega^{+} \text {, the conditions } \\ u \in C_{f}^{\infty}\left(\Omega^{+}\right) \text {and } P u=0 \text { in } \Gamma \text { imply that } u=0 \text { in } \Gamma .\end{array}\right.$ 
Here, $\Gamma_{\mu_{0}}\left(\hat{t}, \hat{x} ; C_{0}\right)=\left\{(t, x) \in \boldsymbol{R}^{n+1} ;|x-\hat{x}| \leqq C_{0}\left(\hat{t}^{\mu_{0}}-t^{\mu_{0}}\right), 0 \leqq t \leqq \hat{t}\right\}$.

When $\mu_{0}=1$, the condition (U) is the existence of a finite propagation speed and when $\mu_{0}<1$, (U) is a weaker condition.

Remark 1.2. (i) The non-characteristic Cauchy problem for operators with $C^{\infty}$-coefficients can be reduced to the flat Cauchy problem as follows. By means of $P u=f$ and $D_{t}^{j} u(0, x)=g_{j}(x)(j=0, \cdots, m-1)$, we can determine the Taylor expansion of $u$ with respect to $t$ at $t=0$. We take a $C^{\infty}$-function $v$ with this Taylor expansion. Then, by the definition of $v, g=f-P v$ is flat at $t=0$. Thus, by putting $\tilde{u}=u-v$, the Cauchy problem " $P u=f$ on $[0, T] \times \boldsymbol{R}^{n}, D_{t}^{j} u(0, x)=$ $g_{j}(x)(j=0, \cdots, m-1)$ " is reduced to the flat Cauchy problem " $P \tilde{u}=g$ on $[0, T]$ $\times \boldsymbol{R}^{n}, \tilde{u}$ is flat at $t=0 "$.

(ii) If the flat Cauchy problem for $P$ is $\left(\mu_{0}\right)$-well-posed at the origin, then $P_{m}(t, x ; \tau, \xi)$ is hyperbolic in a neighborhood of the origin when $t>0$. That is, there exist a positive number $\varepsilon$ and a neighborhood $U$ of $x=0$ such that the equation $P_{m}(t, x ; \tau, \xi)=0$ has only real roots with respect to $\tau$, when $(t, x ; \xi)$ $\in(0, \varepsilon) \times U \times \boldsymbol{R}^{n}$. This follows from the fact that for sufficiently small $t_{0}>0$, the Cauchy problem for $P$ with the initial surface $t=t_{0}$ is $C^{\infty}$-well-posed.

Now, for simplicity, we consider the direction $\left(0, e_{n}\right)=(0,0, \cdots, 0,1) \in \mathbb{R}^{n+1}$. We define

$\mu_{j, \alpha^{\prime}}^{(h)}=\sup \left\{\mu \in \boldsymbol{R}\right.$; there exist a neighborhood $\Omega$ of the origin and $g \in E_{M}\left(\Omega^{+}\right)$ such that $\partial_{\tau}^{j} \partial_{\xi^{\prime}}^{\alpha^{\prime}} P_{m-h}\left(t, x ; 0, e_{n}\right)=t^{\mu} g(t, x)$ in $\left.\Omega^{+}\right\} \in \boldsymbol{R} \cup\{\infty\}$,

where $h=0, \cdots, m$ and $j+\left|\alpha^{\prime}\right| \leqq m-h$. We plot the points $\left(j, \mu_{j, 0}^{(0)}\right)(j=0, \cdots, m)$, and draw the Newton polygon $\pi$ in $(j, \mu)$-plane. We will review on the Newton polygon. We define $\nu(\kappa)=\inf _{0 \leqq j \leqq m}\left(\mu_{j, 0}^{(0)}-\kappa j\right)$ for a real number $\kappa$ and $\mu(j)=$ $\sup \{\kappa j+\nu(\kappa) ; \kappa \in \boldsymbol{R}\}$. Put $\hat{j}=\max \left\{j ; \mu_{k, 0}^{(0)}=\infty\right.$ for $\left.k<j\right\}$. Then, we have

$$
\mu(j)=\infty \quad \text { if } j<\hat{j}, \quad \mu(j)<\infty \text { if } j \geqq \hat{j} .
$$

We call $\mathscr{\Omega}=\{(j, \mu) \in[0, m] \times(\boldsymbol{R} \cup\{\infty\}) ; \mu \geqq \mu(j)\}$ the Newton polygon drawn by $\left\{\left(j, \mu_{j, 0}^{(0)}\right) ; j=0, \cdots, m\right\}$. The non-vertical sides $\mu=\mu(j)(\hat{j} \leqq j \leqq m)$ consists of a finite number of segments $L_{r} ; \mu=\nu_{r}-\kappa_{r} j\left(j_{r} \leqq j \leqq j_{r-1}\right)$, where $r=1, \cdots, l$ and

$$
\kappa_{1}<\kappa_{2}<\cdots<\kappa_{l}, \quad m \kappa_{1}=\nu_{1}<\nu_{2}<\cdots<\nu_{l}, \quad m=j_{0}>j_{1}>\cdots>j_{l}=\hat{j} .
$$

Note that

$$
\mu\left(j_{r}\right)=\mu_{j_{r}, 0}^{(0)} \quad(r=0, \cdots, l) .
$$

Put $m_{r}=j_{r-1}-j_{r} \quad(r=1, \cdots, l) \quad$ and $\quad \kappa_{1}^{*}=\cdots \kappa_{m_{1}}^{*}=\kappa_{1}, \quad \kappa_{m_{1}+1}^{*}=\cdots \kappa_{m_{1}+m_{2}}^{*}=\kappa_{2}, \cdots$, $\kappa_{m_{1}+\cdots+m_{l-1}+1}^{*}=\cdots=\kappa_{m-\hat{j}}^{*}=\kappa_{l}$. Then, we have

$$
\mu(j)=\sum_{r=1}^{m-j} \kappa_{r}^{*} \quad(j=\hat{j}, \hat{j}+1, \cdots, m) .
$$

Since $\hat{j}$ and $\kappa_{1}^{*}, \cdots, \kappa_{m-\hat{j}}^{*}$ determine $\Re$ completely, we denote $\Re=\Re\left\{\hat{j} ; \kappa_{1}^{*}, \cdots\right.$, $\left.\kappa_{m-\hat{j}}^{*}\right\}$. As for the meaning of $\kappa_{r}^{*}$, see Lemma 3.1 in Section 3. Now, we can 
state our result.

Theorem 1. We assume $\left(A-M, \mu_{0}\right)$ and that the flat Cauchy problem for $P$ is $\left(\mu_{0}\right)$-well-posed at the origin. Then,

$$
\left(j+\left|\alpha^{\prime}\right|+h, \mu_{j, \alpha^{\prime}}^{(h)}+h+\left(1-\mu_{0}\right)\left|\alpha^{\prime}\right|\right) \in \Re \quad \text { for any }\left(j, \alpha^{\prime}, h\right) .
$$

Especially, the Newton polygon $\Re_{h}$ drawn by $\left\{\left(j, \mu_{j, 0}^{(h)}\right) ; j=0, \cdots, m-h\right\}$ is contained in the translation of $\Omega$ to the direction $(-h,-h)$.

Next, we pay attention to one side $L_{r} ; \mu=\nu_{r}-\kappa_{r} j=\nu-\kappa j$. If (1.5) holds, then in a neighborhood $\Omega$ of the origin we have

$$
\partial_{\tau}^{j} \partial_{\xi^{\prime}}^{\alpha^{\prime}} P_{m-h}\left(t, x ; 0, e_{n}\right)=j ! \alpha^{\prime} ! t^{\nu-\kappa\left(j+\left|\alpha^{\prime}\right|+h\right)-h+\left(\mu_{0}-1\right) \mid \alpha^{\prime}} b_{j, \alpha^{\prime}}^{(h)}(t, x),
$$

where $b_{j, \alpha^{\prime}}^{(h)}=\boldsymbol{b}_{j, \alpha^{\prime}}^{(h)}\{P ; \nu, \kappa\} \in E_{M}\left(\Omega^{+}\right)$.

We put

$$
\begin{aligned}
f_{\alpha^{\prime}}^{(h)}(\tau) & =\boldsymbol{f}_{\alpha^{\prime}}^{(h)}\left\{b_{j, \alpha^{\prime}}^{(h)} ; j=0, \cdots, m-h-\left|\alpha^{\prime}\right|\right\}(\tau) \\
& =\sum_{j=0}^{m-h-\left|\alpha^{\prime}\right|} b_{j, \alpha^{\prime}}^{(h)}(0,0) \tau^{j}
\end{aligned}
$$

and take a root $\tau_{0}$ of $f_{0}^{(0)}(\tau)=0$ with multiplicity $d$. Put

$$
\begin{aligned}
B_{j, \alpha^{\prime}}^{(h)}= & \mathbb{B}_{j, \alpha^{\prime}}^{(h)}\left\{\tau_{0} ; f_{\alpha^{\prime}}^{(s)}, s=0, \cdots, h\right\} \\
= & \frac{1}{j !} \sum_{s=0}^{h} \sum_{\mu=0}^{h-s} \sum_{g \in J(h, s, \mu)} \frac{\tau_{0}^{\mu}}{\mu ! g_{1} ! \cdots g_{\mu} ! i^{h-s}} \\
& \times \partial_{\tau}^{j+h-s+\mu} f_{\alpha^{\prime}}^{(s)}\left(\tau_{0}\right) \prod_{\theta=1}^{\mu}\left\{\kappa(\kappa-1) \cdots\left(\kappa-g_{\theta}+2\right)\right\},
\end{aligned}
$$

where $J(h, s, \mu)=\left\{g=\left(g_{1}, \cdots, g_{\mu}\right) ; g_{\theta}\right.$ is an integer, $g_{\theta} \geqq 2$ and $g_{1}+\cdots+g_{\mu}=$ $h-s+\mu\}$.

Theorem 2. Under the assumption of Theorem 1, we have

$$
B_{j, \alpha^{\prime}}^{(h)}=0 \quad \text { if } h+j+\left|\alpha^{\prime}\right| \leqq d-1 .
$$

Note that $B_{d, 0}^{(0)}=\frac{1}{d !} \partial_{\tau}^{d} f_{0}^{(0)}\left(\tau_{0}\right) \neq 0$.

Finally we take the roots $\lambda_{1}, \cdots, \lambda_{d}$ of

$$
\sum_{h=0}^{d} B_{d-h, 0}^{(h)} \lambda\left(\lambda-\frac{1}{i}\right) \cdots\left(\lambda-\frac{d-h-1}{i}\right)=0 .
$$

The following theorem is an extention of Theorem 3.3 in [3].

Theorem 3. We assume $\left(A-M, \mu_{0}\right),(1.5),(1.7)$. Then, there exists a constant $C$ which depends only on $M, \mu_{0}, m, \nu$ and $\kappa$ such that the energy inequality (1.10) implies

$$
\begin{gathered}
\max _{h}\left(-\operatorname{Im} \lambda_{h}\right) \leqq C(q+m-p) . \\
\|u\|_{p, \theta} \leqq C^{\prime}\|P u\|_{q, \theta}
\end{gathered}
$$


for any $u \in C_{0}^{\infty}\left(\Omega^{+}\right)$and any $\theta \in[0, T]$, where $T>0, \Omega$ is a neighborhood of the origin, $p, q$ are integers, $C^{\prime}$ is a constant independent of $u$ and $\theta,\|\cdot\|_{p, \theta}$ denotes the Sobolev norm of order $p$ on $\Omega_{\theta}^{+}$and $C_{0}^{\infty}\left(\Omega^{+}\right)=\left\{f \in C_{0}^{\infty}\left(\mathbb{R}^{n+1}\right)\right.$; $\left.\operatorname{supp} f \subset \Omega^{+}\right\}$.

Remark 1.3. (i) In Theorem 1 and 2 , main interest lies in the case $\alpha^{\prime}=0$.

(ii) As for sufficient conditions, there exist many references. Here, we only note [4], [5]. (See also the references of [3].) We can prove the necessity of Sakamoto's conditions in [4] under the assumption $\left(A-M, \mu_{0}\right)$, as a corollary of Theorem 1 .

(iii) As for the meaning of $B_{j, \alpha^{\prime}}^{(h)}$, see Proposition 2.4. Here, we only note that $B_{j, a^{\prime}}^{(h)}=b_{j, \alpha^{\prime}}^{(h)}(0,0)$ if $\tau_{0}=0 . B_{j, \alpha^{\prime}}^{(h)}$ for $h=0,1$ are as follows.

$$
\begin{aligned}
& B_{j, \alpha^{\prime}}^{(0)}=\frac{1}{j !} \partial_{\tau}^{j} f_{a^{\prime}}^{(0)}\left(\tau_{0}\right), \\
& B_{j, \alpha^{\prime}}^{(1)}=\frac{1}{j !}\left\{\frac{\tau_{0} \kappa}{2 i} \partial_{\tau}^{j+2} f_{\alpha^{\prime}}^{(0)}\left(\tau_{0}\right)+\partial_{\tau}^{j} f_{\alpha^{\prime}}^{(1)}\left(\tau_{0}\right)\right\} .
\end{aligned}
$$

(iv) In Theorem 3 , if (1.10) holds, then $q+m-p \geqq 1$.

We will give some examples. In the following examples, $n=1$ and $\kappa$ 's are rational numbers.

\section{Example 1.}

$$
\begin{aligned}
P_{m}=P_{3} & =\left(\tau-t^{\kappa_{1}} \xi\right)\left(\tau-t^{\kappa_{2}} \xi\right)\left(\tau-t^{\kappa_{3}} \xi\right) \\
& =\tau^{3}-\left(t^{\kappa_{1}}+t^{\kappa_{2}}+t^{\kappa_{3}}\right) \tau^{2} \xi+\left(t^{\kappa_{1}+\kappa_{2}}+t^{\kappa_{1}+\kappa_{3}}+t^{\kappa_{2}+\kappa_{3}}\right) \tau \xi^{2}-t^{\kappa_{1}+\kappa_{2}+\kappa_{3}} \xi^{3},
\end{aligned}
$$

where $\kappa_{3}>\kappa_{2}>\kappa_{1}>-1$.

Then, (1.5) claims that the lower order terms have the following forms:

$$
\left(\begin{array}{l}
P_{2}=A t^{-1} \tau^{2}+B t^{\kappa_{1}-1} \tau \xi+C t^{\kappa_{1}+\kappa_{2}-1} \xi^{2}, \\
P_{1}=D t^{-2} \tau+E t^{\kappa_{1}-2} \xi, \\
P_{0}=F t^{-3},
\end{array}\right.
$$

where $A, B, \cdots, F \in E([0, T] \times \boldsymbol{R})$. For this operator, it is already known that the flat Cauchy problem is $\left(\kappa_{1}+1\right)$-well-posed. (See [4].) In this case, the result of Theorem 2 is included in Theorem 1, and the equations (1.8) are

$$
\left(\begin{array}{l}
-\lambda\left(\lambda-\frac{1}{i}\right)+B(0,0) \lambda+E(0,0)=0, \\
\lambda+A(0,0)+B(0,0)+\frac{2 \kappa_{1}}{i}=0, \\
\lambda+C(0,0)=0, \\
-\lambda-\frac{\kappa_{2}}{i}+B(0,0)+C(0,0)=0 .
\end{array}\right.
$$

\section{Example 2.}

$$
\begin{aligned}
P_{4} & =\left(\tau-t^{\kappa_{1}} \xi\right)\left(\tau-t^{\kappa_{2}} \xi\right)\left\{\tau-\left(t^{\kappa_{1}}+t^{\kappa_{2}}\right) \xi\right\} \tau \\
& =\tau^{4}-2\left(t^{\kappa_{1}}+t^{\kappa_{2}}\right) \tau^{3} \xi+\left(t^{2 \kappa_{1}}+3 t^{\kappa_{1}+\kappa_{2}}+t^{2 \kappa_{2}}\right) \tau^{2} \xi^{2}-\left(t^{2 \kappa_{1}+\kappa_{2}}+t^{\kappa_{1}+2 \kappa_{2}}\right) \tau \xi^{3},
\end{aligned}
$$


where $\kappa_{2}>\kappa_{1}>-1$.

Then, (1.5) claims that the lower order terms have the following forms:

$$
\left(\begin{array}{l}
P_{3}=A t^{-1} \tau^{3}+B t^{\kappa_{1}-1} \tau^{2} \xi+C t^{2 \kappa_{1}-1} \tau \xi^{2}+D t^{2 \kappa_{1}+\kappa_{2}-1} \xi^{3}, \\
P_{2}=E t^{-2} \tau^{2}+F t^{\kappa_{1}-2} \tau \xi+G t^{2 \kappa_{1}-2} \xi^{2}, \\
P_{1}=H t^{-3} \tau+I t^{\kappa_{1}-3} \xi \\
P_{0}=J t^{-4}
\end{array}\right.
$$

where $A, B, \cdots, J \in E([0, T] \times \boldsymbol{R})$. In this case, Theorem 2 claims that

$$
A(0,0)+B(0,0)+C(0,0)+\frac{\kappa_{1}}{i}=0 \text {. }
$$

And the equations (1.8) are

$$
\left(\begin{array}{l}
\lambda\left(\lambda-\frac{1}{i}\right)+C(0,0) \lambda+G(0,0)=0, \\
\lambda\left(\lambda-\frac{1}{i}\right)+\alpha \lambda+\beta=0, \\
\text { where } \alpha=3 A(0,0)+2 B(0,0)+C(0,0)+\frac{6 \kappa_{1}}{i} \text { and } \\
\beta=\frac{\kappa_{1}}{i}\{3 A(0,0)+B(0,0)\}+E(0,0)+F(0,0)+G(0,0)-8 \kappa_{1}^{2}+2 \kappa_{1}, \\
-\lambda+D(0,0)=0, \\
\lambda+C(0,0)+D(0,0)+\frac{\kappa_{2}}{i}=0 .
\end{array}\right.
$$

Further, Theorem 2 can be applied to any $x \in \mathbb{R}$, so if we put

$$
R(t, x)=A(t, x)+B(t, x)+C(t, x)+\frac{\kappa_{1}}{i},
$$

then we have $R(0, x) \equiv 0$. This means that there exist a positive number $\varepsilon$ and $S \in E([0, T] \times \boldsymbol{R})$ such that

$$
R(t, x)=t^{\varepsilon} S(t, x) .
$$

As a matter of fact, by coordinate transformation and Theorem 1 , we can prove that the following condition is necessary for well-posedness;

$$
R(t, x) \in t^{\kappa_{2}-\kappa_{1}} \times E([0, T] \times \boldsymbol{R}) .
$$

And it can be also proved that this condition is safficient.

\section{$\S 2$. Two Transformations of Operators}

In this and the next sections, we state some preliminary results which are necessary to prove the theorems.

First, for a positive real number $p$, we define $T_{p} ; C_{f}^{\infty}\left(\Omega^{+}\right) \rightarrow C_{f}^{\infty}\left(\widetilde{\Omega}^{+}\right)$, where $\Omega=\left\{(t, x) ;\left(t^{1 / p}, x\right) \in \Omega\right\}$, by

$$
\left(T_{p} u\right)(t, x)=u\left(t^{1 / p}, x\right) .
$$

The next lemma is almost trivial. So the proof is omitted. 
Lemma 2.1. $T_{p}$ satisfies the following properties.

(i) $T_{p}^{-1}=T_{p^{-1}}$.

(ii) $T_{p}^{-1} \circ \partial_{t} \circ T_{p}=\frac{1}{p} t^{1-p} \partial_{t}$, where $P \circ Q$ denotes the composition of operators $P$ and $Q$.

(iii) $T_{p}^{-1} \circ a(t, x) \circ T_{p}=a\left(t^{p}, x\right)$, where $a(t, x)$ is considered as a multiplication operator.

(iv) For any positive integer $j$, there exist constants $e_{k}^{(j)}(k=1, \cdots, j)$ such that $e_{j}^{(j)}=1$ and

$$
T_{p}^{-1} \circ \partial_{t}^{j} \circ T_{p}=p^{-j} \sum_{k=1}^{j} e_{k}^{(j)} t^{k-p j} \partial_{t}^{k}
$$

For a differential operator $P$ and a positive rational number $p$, we put $\mathscr{I}_{p}(P)=p^{m} t^{m(p-1)} T_{p}^{-1} \odot P \circ T_{p}$.

Proposition 2.2. (i) If $P$ satisfies $\left(A-M, \mu_{0}\right)$, then $\tilde{P}=\mathscr{I}_{p}(P)$ satisfies $\left(A-\tilde{M}, \tilde{\mu}_{0}\right)$ with $\tilde{\mu}_{0}=p \mu_{0}, \tilde{M}=\frac{M}{p} n$, where $n$ is a positive integer such that $\tilde{M}$ is an integer.

(ii) If the flat Cauchy problem for $P$ is $\left(\mu_{0}\right)$-well-posed at the origin, then the flat Cauchy problem for $\tilde{P}$ is $\left(\tilde{\mu}_{0}\right)$-well-posed at the origin.

(iii) If

$$
\partial_{\tau}^{j} \partial_{\xi^{\prime}}^{\alpha^{\prime}} P_{m-h}\left(t, \hat{x} ; 0, e_{n}\right)=t^{\nu-\kappa\left(j+\left|\alpha^{\prime}\right|+h\right)-h+\left(\mu_{0}-1\right)\left|a^{\prime}\right|} d_{j, \alpha^{\prime}}^{(h)}(t),
$$

where $d_{j, \alpha^{\prime}}^{(h)} \in C^{0}[0, T]$, then

$$
\partial_{\tau}^{j} \partial_{\xi^{\prime}}^{\alpha^{\prime}} \widetilde{P}_{m-h}\left(t, \hat{x} ; 0, e_{n}\right)=t^{\tilde{\nu}-\tilde{\kappa}\left(j+\left|\alpha^{\prime}\right|+h\right)-h+\left(\tilde{\mu}_{0}-1\right)\left|\alpha^{\prime}\right|} \tilde{d}_{j, \alpha^{\prime}}^{(h)}(t),
$$

where $\tilde{d}_{j_{,}, \alpha^{\prime}}^{(h)} \in C^{0}\left[0, T^{1 / p}\right], \tilde{\nu}=p \nu+(p-1) m$ and $\tilde{\kappa}=p \kappa+p-1$. Further, $\tilde{d}_{j^{\prime}, \alpha^{\prime}}^{(0)}(t)=$ $p^{m-j} d_{j, \alpha^{\prime}}^{(0)}\left(t^{p}\right)$. And for fixed $\alpha^{\prime}$ and $d$, if $d_{j, \alpha^{\prime}}^{(h)}(0)=0$ for $h+j<d$, then $\tilde{d}_{j, \alpha^{\prime}}^{(h)}(0)$ $=0$ for $h+j<d$.

(iv) $\mathscr{I}_{p^{-1}}\left(I_{p}(P)\right)=I_{p}\left(I_{p^{-1}}(P)\right)=P$.

Proof. We compute $\tilde{P}$ by means of (iii), (iv) of Lemma 2.1. If

$$
P=\sum_{j+\alpha \mid \leqslant m} a_{j, \alpha}(t, x) D_{t}^{j} D_{x}^{a},
$$

then

$$
\tilde{P}=\sum_{k+|\alpha| \leqq m} \tilde{a}_{k, \alpha}(t, x) D_{t}^{k} D_{x}^{\alpha}
$$

where

$$
\tilde{a}_{k, \alpha}(t, x)=\sum_{j=k}^{m-|\alpha|} a_{j, \alpha}\left(t^{p}, x\right) p^{m-j} e_{k}^{(j)} t^{k-m+p(m-j)} .
$$

So, (i) is trivial. If

then

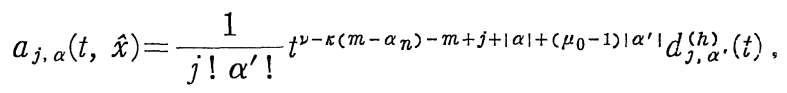


So, (2.2) holds and

$$
\begin{aligned}
& \tilde{a}_{k, \alpha}(t, \hat{x})=\frac{1}{\alpha^{\prime} !} t^{\tilde{\nu}-\tilde{\kappa}(m-\alpha n)-m+k+|\alpha|+\left(\tilde{\mu}_{0}-1\right)\left|\alpha^{\prime}\right|} \\
& \times \sum_{j=k}^{m-|\alpha|} \frac{1}{j !} p^{m-j} e_{k}^{(j)} d_{j, \alpha^{\prime}}^{(m-j-|\alpha|)}\left(t^{p}\right) .
\end{aligned}
$$

$$
\tilde{d}_{k, \alpha^{\prime}}^{(h)}(t)=\sum_{j=k}^{k+h} \frac{k !}{j !} p^{m-j} e_{k}^{(j)} d_{j, \alpha^{\prime}}^{(h+k-j)}\left(t^{p}\right) .
$$

(iii) follows from this. To prove (ii), we have only to check the condition $(U)$. If $f(t, x)=0$ in $\Gamma_{\tilde{\mu}_{0}}\left(\hat{t}^{1 / p}, \hat{x} ; C_{0}\right)$, then $\left(T_{p} f\right)(t, x)=f\left(t^{1 / p}, x\right)=0$ in

$$
\begin{aligned}
& \left\{(t, x) \in \mathbb{R}^{n+1} ;|x-\hat{x}| \leqq C_{0}\left\{\left(\hat{t}^{1 / p}\right)^{\tilde{\mu}_{0}}-\left(t^{1 / p}\right)^{\tilde{\mu}_{0}}\right\}, 0 \leqq t^{1 / p} \leqq \hat{t}^{1 / p}\right\} \\
& =\Gamma_{\mu_{0}}\left(\hat{t}, \hat{x} ; C_{0}\right) .
\end{aligned}
$$

And conversely, if $\left(T_{p} u\right)(t, x)=0$ in $\Gamma_{\mu_{0}}\left(\hat{t}, \hat{x} ; C_{0}\right)$, we can prove that $u(t, x)=0$ in $\Gamma_{\tilde{\mu}_{0}}\left(\hat{t}^{1 / p}, \hat{x} ; C_{0}\right)$. Thus, (ii) is proved. (iv) is trivial.

Remark 2.3. If $\Omega=\{(j, \mu) ; \mu \geqq \mu(j)\}$ is the Newton polygon drawn from $P$, then the Newton polygon drawn from $\widetilde{P}=\mathscr{I}_{p}(P)$ is

$$
\tilde{n}=\{(j, \mu) ; \mu \geqq p \mu(j)+(p-1)(m-j)\} .
$$

Next, assume that $P$ satisfies $\left(A-M, \mu_{0}\right)$ and (1.6). Define $f_{\alpha^{\prime}}^{(h)}$, take a root $\tau_{0}$ with multiplicity $d$ and define $B_{j, \alpha^{\prime}}^{(h)}$ as in Section 1 . And perform the following coordinate transformation in a neighborhood $\Omega$ of the origin:

$$
\left\{\begin{array}{l}
s=t, \\
y_{j}=x_{j}+t^{\mu_{0}+\varepsilon} f_{j}(t, x) \quad(j=1, \cdots, n-1), \\
y_{n}=x_{n}+\frac{1}{\kappa+1} t^{\kappa+1} \tilde{\tau}(t, x),
\end{array}\right.
$$

where $\varepsilon$ is a positive number such that $\varepsilon M$ is an integer and $f_{j}(j=1, \cdots, n-1)$, $\tilde{\tau} \in E_{M}\left(\Omega^{+}\right)$. Let $P\left(t, x ; D_{t}, D_{x}\right)$ be transformed into $\widetilde{P}\left(s, y ; D_{s}, D_{y}\right)$. Then, the following proposition holds.

Proposition 2.4. $\tilde{P}$ satisfies $\left(A-M, \mu_{0}\right)$ and there exist $\tilde{b}_{j, \alpha^{\prime}}^{(h)} \in E_{M}\left(\Omega^{+}\right)$such that there holds

!

We put $\tilde{f}_{\alpha^{\prime}}^{(h)}=f_{\alpha^{\prime}}^{(h)}\left\{\tilde{b}_{j, \alpha^{\prime}}^{(h)} ; j=0, \cdots, m-h-\left|\alpha^{\prime}\right|\right\}$, then $\sigma_{0}=\tau_{0}-\tilde{\tau}(0,0)$ is a root of $\tilde{f}_{0}^{(0)}(\sigma)=0$ with multiplicity $d$. We put $\tilde{B}_{j, \alpha^{\prime}}^{(h)}=\boldsymbol{B}_{j, \alpha^{\prime}}^{(h)}\left\{\sigma_{0} ; \tilde{f}_{\alpha^{\prime}}^{(s)}, s=0, \cdots, h\right\}$. Then,

$$
B_{j, \alpha^{\prime}}^{(h)}=\tilde{B}_{j, \alpha^{\prime}}^{(h)} \quad\left(h=0, \cdots, m ; j+\left|\alpha^{\prime}\right| \leqq m-h\right) .
$$

Especially, if $\tilde{\tau}(0,0)=\tau_{0}$, then $\tilde{b}_{j, \alpha^{\prime}}^{(h)}(0,0)=B_{j, \alpha^{\prime}}^{(h)}$.

Proof. By a straight computation of $\tilde{P}$ we get (2.4) and

$$
\tilde{b}_{j, \alpha^{\prime}}^{(h)}(0,0)=\frac{1}{j !} \sum_{k=0}^{h} \sum_{\mu=0}^{h-k} \sum_{g \in J(h, k, \mu)} \frac{\tilde{\tau}(0,0)^{\mu} i^{s-h}}{\mu ! g_{1} ! \cdots g_{\mu} !}
$$




$$
\times\left(\partial_{\tau}^{j+h-s+\mu} f_{\alpha^{\prime}}^{(s)}\right)(\widetilde{\tau}(0,0)) \prod_{\theta=1}^{\mu}\left\{\kappa(\kappa-1) \cdots\left(\kappa-g_{\theta}+2\right)\right\} .
$$

This shows that $\tilde{b}_{j, \alpha^{\prime}}^{(h)}(0,0)$ depends only on $\tilde{\tau}(0,0)$ as for the coordinate transformation. Further,

$$
\left(\begin{array}{l}
\tilde{f}_{0}^{(0)}(\sigma)=f_{0}^{(0)}(\sigma+\tilde{\tau}(0,0)), \\
\tilde{b}_{j, a^{\prime}}^{(h)}(0,0)=B_{j, a^{\prime}}^{(h)} \quad \text { if } \quad \tilde{\tau}(0,0)=\tau_{0} .
\end{array}\right.
$$

Now, we consider another coordinate transformation;

$$
\left\{\begin{array}{l}
u=s(=t), \\
w_{j}=y_{j}\left(=x_{j}+t^{\mu_{0}+\varepsilon} f_{\jmath}(t, x)\right) \quad(j=1, \cdots, n-1), \\
w_{n}=y_{n}+\frac{1}{\kappa+1} s^{\kappa+1}\left(\tau_{0}-\tilde{\tau}(0,0)\right) \\
\quad\left(=x_{n}+\frac{1}{\kappa+1} t^{\kappa+1}\left(\tilde{\tau}(t, x)-\tilde{\tau}(0,0)+\tau_{0}\right)\right) .
\end{array}\right.
$$

Let $\underset{P}{\tilde{P}}$ be transformed into $\tilde{\widetilde{P}}\left(u, w ; D_{u}, D_{w}\right)$ and put $\widetilde{\widetilde{b}}_{j, \alpha^{\prime}}^{(h)}=\widetilde{b}_{j, \alpha^{\prime}}^{(h)}\{\tilde{\widetilde{P}} ; \nu, \kappa\}, \tilde{\widetilde{f}}_{\alpha^{\prime}}^{(h)}=$ $\boldsymbol{f}_{\alpha^{\prime}}^{(h)}\left\{\widetilde{\tilde{b}}_{j, \alpha^{\prime}}^{(h)} ; j=0, \cdots, m-h-\left|\alpha^{\prime}\right|\right\}$. Then, $\lambda=0$ is a root of $\widetilde{\widetilde{f}}_{0}^{(0)}(\lambda)=0$ with multiplicity $d$. Applying (2.6) for $P$ and $\widetilde{\widetilde{P}}$, we have $\widetilde{\widetilde{b}}_{j, \alpha^{\prime}}^{(h)}(0,0)=B_{j, \alpha^{\prime}}^{(h)}$, and applying (2.6) for $\tilde{P}$ and $\widetilde{\widetilde{P}}$, we have $\widetilde{\widetilde{b}}_{j, a^{\prime}}^{(h)}(0,0)=\widetilde{B}_{j, \alpha^{\prime}}^{(h)}$. So, we get the desired result.

\section{$\S 3$. Some Remarks on the Newton Polygon}

We define

$$
\begin{aligned}
& F_{r}=\left\{t^{r} f(t) ; f \in C^{0}[0, T], f(0) \neq 0\right\} \quad(r \in \mathbb{R}), \\
& F_{\infty}=\left\{f \in C^{0}[0, T] ; \text { for any } r \in \mathbb{R}, t^{-r} f(t) \in C^{0}[0, T]\right\} .
\end{aligned}
$$

The following lemma states the meaning of the Newton polygon.

Lemma 3.1. We consider $f(t, \tau)=\sum_{j=0}^{m} a_{j}(t) \tau^{j}$, where $a_{j} \in F_{\mu_{j}}, \mu_{j} \in \mathbb{R} \cup\{\infty\}$ $(j=0, \cdots, m)$ and $a_{m}(t) \equiv 1$. We draw the Newton polygon $\Re_{1}$ by $\left\{\left(j, \mu_{j}\right) ; j=0\right.$, $\cdots, m\}$ and get $\hat{j}, \kappa_{1}^{*}, \cdots, \kappa_{m-\hat{j}}^{*}$ as in Section 1. Then, there exist $\sigma_{r} \in F_{\kappa_{r}^{*}}(r=1, \cdots$, $m-\hat{j})$ and $\sigma_{r} \in F_{\infty}(r=m-\hat{j}+1, \cdots, m)$ such that

$$
f(t, \tau)=\prod_{r=1}^{m}\left(\tau-\sigma_{r}(t)\right) .
$$

Proof. We can determine $\kappa_{1}<\cdots<\kappa_{l}, m \kappa_{1}=\nu_{1}<\cdots<\nu_{l}, m=j_{0}>j_{1}>\cdots>j_{l}=\hat{j}$ as in Section 1. We will prove this lemma by induction on $l$. If $l=0$, then $\hat{j}=m$ and $a_{j} \in F_{\infty}(j=0, \cdots, m-1)$. For any $\kappa \in \boldsymbol{R}$, the polynomial

$$
f_{1}(t, \tau)=\tau^{-\kappa m} f\left(t, t^{\kappa} \tau\right)=\sum_{j=0}^{m} t^{\kappa(j-m)} a_{\jmath}(t) \tau^{j}
$$

has continuous coefficients. On the other hand, there exist $\sigma_{r} \in C^{0}[0, T](r=1$, $\cdots, m)$ such that $f(t, \tau)=\prod_{r=1}^{m}\left(\tau-\sigma_{r}(t)\right)$ and so, $f_{1}(t, \tau)=\prod_{r=1}^{m}\left(\tau-t^{-\kappa} \sigma_{r}(t)\right)$. Thus, we 
have $\sigma_{r} \in F_{\infty}$.

Next, we assume that $l \geqq 1$ and the lemma holds for $l-1$. We consider

$$
\begin{aligned}
f_{1}(t, \tau)=t^{-\nu_{1}} f\left(t, t^{\kappa_{1}} \tau\right) & =\sum_{j=0}^{m} a_{j}(t) t^{\kappa_{1} j-\nu_{1}} \tau^{j} \\
& =\sum_{j=0}^{m} b_{j}(t) \tau^{j} .
\end{aligned}
$$

By the definition of $\nu_{1}, \kappa_{1}$, we have $b_{j} \in C^{0}[0, T](j=0, \cdots, m), b_{m}(t) \equiv 1$ and $b_{j_{1}}(0) \neq 0$. So, there exist $\hat{\sigma}_{k} \in F_{0}\left(k=1, \cdots, m_{1}=m-j_{1}\right)$ and a monic polynomial $g_{1}(t, \tau)$ of degree $j_{1}$ with $C^{0}$-coefficients such that

$$
f_{1}(t, \tau)=\prod_{k=1}^{m_{1}}\left(\tau-\hat{\sigma}_{k}(t)\right) g_{1}(t, \tau)
$$

So, we have

$$
f(t, \tau)=t^{m_{1}} f_{1}\left(t, t^{-\kappa_{1}} \tau\right)=\prod_{k=1}^{m_{1}}\left(\tau-t^{\kappa_{1}} \hat{\sigma}_{k}(t)\right) g(t, \tau),
$$

where $g(t, \tau)=\sum_{j=0}^{j_{1}} d_{j}(t) \tau^{j}$ is a monic polynomial of degree $j_{1}$ and $d_{j} \in C^{0}(0, T]$ $\left(j=0, \cdots, j_{1}\right)$. We claim that

$$
d_{j} \in F_{\mu_{j}-\kappa_{1} m_{1}} \quad\left(j=0, \cdots, j_{1}\right)
$$

Set

$$
\prod_{k=1}^{m_{1}}\left(\tau-t^{\kappa_{1}} \hat{\sigma}_{k}(t)\right)=\sum_{j=0}^{m_{1}} t^{\kappa_{1}\left(m_{1}-j\right)} e_{j}(t) \tau^{j},
$$

where $e_{j} \in C^{0}[0, T], e_{m_{1}}(t) \equiv 1$ and $e_{0}(0) \neq 0$. Then, by (3.3),

$$
a_{\jmath}(t)=\sum_{p+q=j} t^{\kappa_{1}\left(m_{1}-p\right)} e_{p}(t) d_{q}(t),
$$

where $j=0, \cdots, j_{1}$.

From (3-6), we have $a_{0}(t)=t^{\kappa_{1} m_{1}} e_{0}(t) d_{0}(t)$. Since $e_{0}(0) \neq 0$, we have $d_{0}(t)=$ $t^{-\kappa_{1} m_{1}} a_{0}(t) e_{0}(t)^{-1} \in F_{\mu_{0}-\kappa_{1} m_{1}}$.

Assume that $0 \leqq h \leqq j_{1}-1$ and (3.4) holds for $j \leqq h$. From (3.6) $)_{h+1}$, we have

$$
t^{\kappa_{1} m_{1}} e_{0}(t) d_{h+1}(t)=a_{h+1}(t)-\sum_{\substack{p+q=h+1 \\ p \geq 1}} t^{\kappa_{1}\left(m_{1}-p\right)} e_{p}(t) d_{q}(t) \text {. }
$$

Now, $e_{p}(t) d_{q}(t) \in t^{\mu_{q}-\kappa_{1} m_{1}} \times C^{0}[0, T]$ and

$$
\mu_{q}>\mu_{h+1}+\kappa_{1}(h+1-q) \quad(q=0, \cdots, h) .
$$

So, we have $d_{h+1} \in F_{\mu_{h+1}-\kappa_{1} m_{1}}$.

Thus, we have proved (3.4). And this means that the Newton polygon drawn from $g$ is determined by $\hat{j}$ and $\kappa_{m_{1}+1}^{*}, \cdots, \kappa_{m-\hat{\jmath}}^{*}$. So, by induction hypothesis, we get the desired result.

The following proposition will be used to prove the case $h=0$ in Theorem 1 and 2. We fix $\hat{x} \in \boldsymbol{R}^{n}$.

Proposition 3.2. We assume $\left(A-M, \mu_{0}\right)$ and 


$$
\partial_{\tau}^{j} P_{m}\left(t, \hat{x} ; 0, e_{n}\right) \in t^{\nu-\kappa j} \times C^{0}[0, T] \quad(j=0, \cdots, m) .
$$

If $P_{m}$ is hyperbolic when $t>0$, then we have

$$
\partial_{\tau}^{j} \partial_{\xi}^{\alpha} P_{m}\left(t, \hat{x} ; 0, e_{n}\right) \in t^{\nu-\kappa(j+|\alpha|)+\left(\mu_{0}-1\right)|a|} \times C^{0}[0, T],
$$

where $j+|\alpha| \leqq m$.

Proof. For $\eta \in S^{n-1}=\left\{\eta \in \mathbb{R}^{n} ;|\eta|=1\right\}$ and $\rho \in \mathbb{R}$, we consider the equation

$$
P_{m}\left(t, \hat{x} ; \tau, e_{n}+\rho \eta\right)=0 \text {. }
$$

For fixed $(t, \eta)$, the left-hand side is a polynomial with respect to $(\rho, \tau)$ and hyperbolic with respect to $\tau$. So, there exist $m$ roots $\tau_{\jmath}(t, \eta ; \rho)$ of $(3.7)$ which are analytic with respect to $\rho$. By $\left(A-M, \mu_{0}\right)$, we have $\left|\tau_{\jmath}(t, \eta ; \rho)\right| \leqq C t^{\mu_{0}-1}$ near $t=0, \rho=0$. On the other hand, we draw the Newton polygon $\Re_{1}$ from the polynomial $P_{m}\left(t, \hat{x} ; \tau, e_{n}\right)$. Let $\Omega_{1}=\Re\left\{\hat{j} ; \kappa_{1}^{*}, \cdots, \kappa_{m-\hat{j}}^{*}\right\}$. Put $\kappa_{m-\hat{j}+1}^{*}=\cdots=\kappa_{m}^{*}=\hat{\kappa}$, where $\hat{\kappa}$ is sufficiently large. By Lemma 3.1, there exist $\sigma_{j} \in t^{\kappa_{j}^{*}} \times C^{0}[0, T]$ $(j=1, \cdots, m)$ such that

$$
P_{m}\left(t, \hat{x} ; \tau, e_{n}\right)=\prod_{\jmath=1}^{m}\left(\tau-\sigma_{\jmath}(t)\right) .
$$

From the above two facts, we can take $\sigma_{\jmath}(t, \eta ; \rho)(j=1, \cdots, m)$ such that

(a) $P_{m}\left(t, \hat{x} ; \tau, e_{n}+\rho \eta\right)=\prod_{\jmath=1}^{m}\left(\tau-\sigma_{\jmath}(t, \eta ; \rho)\right)$,

(b) for fixed $(t, \eta), \sigma_{j}(t, \eta ; \rho)$ is analytic with respect to $\rho$,

(c) $\left|\sigma_{\jmath}(t, \eta ; 0)\right| \leqq C t^{\kappa_{j}^{*}},\left|\sigma_{j}(t, \eta ; \rho)\right| \leqq C t^{\mu_{0}-1}$, near $t=0$.

By Cauchy's integral formula and (c), we have

(d) $\left|\partial_{\rho}^{k} \sigma_{j}(t, \eta ; \rho)\right| \leqq C t^{\mu_{0}-1}$ for any $k$ and $j$.

So, the $t$-order of $\partial_{\tau}^{j} \partial_{\rho}^{k}\left(P_{m}\left(t, \hat{x} ; \tau, e_{n}+\rho \eta\right)\right)_{\mid \rho=0, \tau=0}$ is not smaller than $K=$ $\sum_{h=1}^{m-j-k} \kappa_{h}^{*}+k\left(\mu_{0}-1\right)$. Since the line $\mu=\nu-\kappa j$ lies below $\Re_{1}$, we have $K \geqq \nu-\kappa(j+k)$ $+\left(\mu_{0}-1\right) k$. So, by choosing $\eta$ in a suitable manner, we obtain the desired result.

\section{$\S 4$. Proof of Theorem 1}

By Proposition 2.2, without loss of generality, we may assume that $M=1$ and that $\mu_{0}, \nu_{r}$ and $\kappa_{r}(r=1, \cdots, l)$ are positive integers and $\mu_{0} \geqq 2$.

Remark 4.1. By the definition of $\Omega$, we have

$$
\partial_{\tau}^{j_{r}} P_{m}\left(t, x ; 0, e_{n}\right)=t^{\mu\left(j_{r}\right)} g_{r}(t, x),
$$

where $g_{r} \in E_{M}\left(\Omega^{+}\right)(r=l, \cdots, l), \Omega$ is a neighborhood of the origin and $g_{r}(0, x)$ $\not \equiv 0$ in any neighborhood of the origin.

Without loss of generality, we may assume the following condition:

(B) $g_{r}(0,0) \neq 0, \quad r=1, \cdots, l$. 
(The proof is given in the appendix.)

We have to prove the followings:

$\left[N_{r}\right]$

$$
\begin{gathered}
\partial_{\tau}^{j} \partial_{\xi^{\prime}}^{\alpha^{\prime}} P_{m-h}\left(t, x ; 0, e_{n}\right) \in t^{\nu} r^{-\kappa_{r}\left(j+\left|\alpha^{\prime}\right|+h\right)-h+\left(\mu_{0}-1\right)\left|\alpha^{\prime}\right|} \times C^{\infty}, \\
h=0, \cdots, m ; j+\left|\alpha^{\prime}\right| \leqq m-h,
\end{gathered}
$$

where $r=1, \cdots, l$, and

$\left[N_{\kappa}\right] \quad \partial_{\tau}^{j} \partial_{\xi^{\prime}}^{\alpha^{\prime}} P_{m-h}\left(t, x ; 0, e_{n}\right) \in t^{\mu j^{+\kappa}-\kappa\left(j+, \alpha^{\prime} \mid+h\right)-h+\left(\mu_{0}-1\right)\left|\alpha^{\prime}\right|} \times C^{\infty}$,

where $\kappa>\kappa_{l}$.

$$
h=0, \cdots, m ; j+\left|\alpha^{\prime}\right| \leqq m-h,
$$

Of course, $\left[N_{k}\right]$ is trivial if $\hat{j}=0$.

For $h=0$, these follows from Proposition 3.2, so we have only to prove the following proposition.

Proposition 4.2. We assume that $\nu, \kappa, \nu^{*}, \kappa^{*}, m^{*}$ and $\varepsilon\left(h, j, \alpha^{\prime}\right)$ are integers and $b_{j, \alpha^{\prime}}^{(h)} \in C^{\infty}\left(\Omega^{+}\right)$, where the followings are satisfied.

(1) $\partial_{\tau}^{j} \partial_{\xi^{\prime}}^{\alpha^{\prime}} P_{m}\left(t, x ; 0, e_{n}\right)=j ! \alpha^{\prime} ! t^{\nu-\kappa\left(j+\left|\alpha^{\prime}\right|\right)+\left(\mu_{0}-1\right)\left|\alpha^{\prime}\right|} \times b_{j, \alpha^{\prime}}^{(0)}(t, x) \quad\left(j+\left|\alpha^{\prime}\right| \leqq m\right)$.

(2) $\partial_{\tau}^{j} \partial_{\xi^{\prime}}^{\alpha^{\prime}} P_{m-h}\left(t, x ; 0, e_{n}\right)=j ! \alpha^{\prime} ! t^{\nu-\kappa\left(j+\left|\alpha^{\prime}\right|+h\right)-h+\left(\mu_{0}-1\right)\left|\alpha^{\prime}\right|-\varepsilon\left(h, j, \alpha^{\prime}\right)} \times b_{j, \alpha^{\prime}}^{(h)}(t, x)$ $\left(h=1, \cdots, m ; j+\left|\alpha^{\prime}\right| \leqq m-h\right)$.

(3) $0<\nu^{*}<\nu, \mu_{0}-1 \leqq \kappa^{*}<\kappa, 0<m^{*} \leqq m, \nu^{*}-\kappa^{*} m^{*}=\nu-\kappa m^{*}$.

(4) $b_{j, \alpha^{\prime}}^{(0)} \in t^{(\kappa *-\kappa)\left(m *-j-\left|\alpha^{\prime}\right|\right)} \times C^{\infty}\left(\Omega^{+}\right)$if $j+\left|\alpha^{\prime}\right|>m^{*}$.

(5) $\quad b_{m *, 0}^{(0)}(0,0) \neq 0$ and $b_{j, \alpha^{\prime}}^{(h)}(0, x) \not \equiv 0$ in any neighborhood of $x=0$ if $\varepsilon\left(h, j, \alpha^{\prime}\right)$ $>0$.

(6) $\varepsilon\left(h, j, \alpha^{\prime}\right) \leqq\left(\kappa-\kappa^{*}\right)\left(m^{*}-h-j-\left|\alpha^{\prime}\right|\right), h=1, \cdots, m ; j+\left|\alpha^{\prime}\right| \leqq m-h$.

If the flat Cauchy problem for $P$ is $\left(\mu_{0}\right)$-well-posed at the origin, then

$$
\varepsilon\left(h, j, \alpha^{\prime}\right) \leqq 0, h=1, \cdots, m ; j+\left|\alpha^{\prime}\right| \leqq m-h .
$$

When $\kappa_{1}=\mu_{0}-1,\left[N_{1}\right]$ is satisfied by $\left(A-M, \mu_{0}\right)$. When $\kappa_{1}>\mu_{0}-1$, we apply Proposition 4.2 with $\kappa=\kappa_{1}, \nu=\nu_{1}=\kappa_{1} m, \kappa^{*}=\mu_{0}-1, \nu^{*}=m\left(\mu_{0}-1\right)$ and $m^{*}=m$. All the assumptions of the proposition is satisfied and so $\left[N_{1}\right]$ follows. We assume $\left[N_{r}\right]$, then the assumptions of Proposition 4.2 are satisfied with $\kappa=\kappa_{r+1}, \nu=\nu_{r+1}$, $\kappa^{*}=\kappa_{r}, \nu^{*}=\nu_{r}, m^{*}=j_{r}$. By Proposition 4.2, we have $\left[N_{r+1}\right]$. In the same manner, if $\left[N_{l}\right]$ holds and $\hat{j}>0$, we have $\left[N_{\kappa}\right]\left(\kappa>\kappa_{l}\right)$ applying Proposition 4.2 with $\nu=\mu_{j}+\kappa \hat{j}, \kappa^{*}=\kappa_{l}, \nu^{*}=\nu_{l}, m^{*}=\hat{j}$. Thus, we get Theorem 1 .

Proof of Proposition 4.2. This proposition is proved by the same method as [2; Theorem 4.1]. (See also [1].) We assume that there exists $\left(h, j, \alpha^{\prime}\right)$ such that $\varepsilon\left(h, j, \alpha^{\prime}\right)>0$. We put

$$
\sigma_{1}=\frac{1}{\kappa-\mu_{0}+2} \max \left\{\frac{\varepsilon\left(h, j, \alpha^{\prime}\right)}{m^{*}-j-\left|\alpha^{\prime}\right|} ; m^{*}-j-\left|\alpha^{\prime}\right|-h>0, h \geqq 1\right\} .
$$

By (6), we have 


$$
0<\sigma_{1}<\frac{\kappa-\kappa^{*}}{\kappa-\mu_{0}+2}(<1)
$$

We define

$$
\mathfrak{M}=\left\{\left(h, j, \alpha^{\prime}\right) ; \sigma_{1}=\frac{1}{\kappa-\mu_{0}+2} \frac{\varepsilon\left(h, j, \alpha^{\prime}\right)}{m^{*}-j-\left|\alpha^{\prime}\right|}, h+j+\left|\alpha^{\prime}\right|<m^{*}, h \geqq 1\right\} .
$$

We may assume that $b_{j, \alpha^{\prime}}^{(h)}(0,0) \neq 0$ for some $\left(h, j, \alpha^{\prime}\right) \in \mathfrak{M}$, without loss of generality. (We translate the origin of $\boldsymbol{R}^{n}$ if necessary.) We put

$$
\mathfrak{M}_{0}=\left\{\left(h, j, \alpha^{\prime}\right) \in \mathfrak{M} ; b_{j, \alpha^{\prime}}^{(h)}(0,0) \neq 0\right\} .
$$

Now, we perform the following coordinate transformation:

$$
\left\{\begin{array}{l}
t=\rho^{-\delta} s, \\
x_{j}=\rho^{-\mu_{0} \delta} y_{j} \quad(j=1, \cdots, n-1), \\
x_{n}=\rho^{-\left(\mu_{0}-1\right) \delta} y_{n}
\end{array}\right.
$$

where $\delta=\left(\kappa-\mu_{0}+2\right)^{-1}$.

By this transformation, $P\left(t, x ; D_{t}, D_{x}\right)$ is transformed into $P_{\rho}\left(s, y ; D_{s}, D_{y}\right)$ which is a differential operator on $B=[0,1] \times B^{\prime}=[0,1] \times\left\{y \in \mathbb{R}^{n} ;|y| \leqq 1\right\}$ for sufficiently large $\rho$. From (1), we have

$$
\begin{aligned}
P_{\rho, m}(s, y ; \sigma, \eta)= & P_{m}\left(\rho^{-\delta} s, \rho^{-\mu_{0} \delta} y^{\prime}, \rho^{-\left(\mu_{0}-1\right) \delta} y_{n} ; \rho^{\delta} \sigma, \rho^{\mu_{0} \delta} \eta^{\prime}, \rho^{\left(\mu_{0}-1\right) \delta} \eta_{n}\right) \\
= & \sum_{j, a^{\prime}} \rho^{-\left\{\nu-\kappa\left(j+\left|\alpha^{\prime}\right|\right)+\left(\mu_{0}-1\right)\left|\alpha^{\prime}\right| \mid \delta \delta\right.} \\
& \times \rho^{\left\{j+\mu_{0}\left|\alpha^{\prime}\right|+\left(\mu_{0}-1\right)\left(m-j-\left|\alpha^{\prime}\right|\right)\right\} \delta} s^{\nu-\kappa\left(j+\left|a^{\prime}\right|\right)+\left(\mu_{0}-1\right)\left|\alpha^{\prime}\right|} \\
& \times b_{j, \alpha^{\prime}}^{(0)}\left(s \rho^{-\delta}, y^{\prime} \rho^{-\mu_{0} \delta}, y_{n} \rho^{-\left(\mu_{0}-1\right) \delta}\right) \sigma^{j} \eta^{\prime a^{\prime}} \eta_{n}^{m-j-\mid \alpha^{\prime}:} \\
= & \sum_{j, \alpha^{\prime}} \rho^{\left\{\left(\kappa-\mu_{0}+2\right)\left(j+\left|\alpha^{\prime}\right|\right)+\left(\mu_{0}-1\right) m-\nu\right\} \delta} \\
& \times s^{\nu-\kappa\left(j+\left|a^{\prime}\right|\right)+\left(\mu_{0}-1\right)\left|\alpha^{\prime}\right|} b_{j, a^{\prime}}^{(0)} \sigma^{j} \eta^{\prime \alpha^{\prime}} \eta_{n}^{m-j-\left|a^{\prime}\right|} .
\end{aligned}
$$

We put $u_{1}=\exp \left\{i \gamma y_{n} \rho+i l^{1}\left(s, y^{\prime}\right) \rho^{\sigma_{1}}\right\}$ where $\gamma$ and $l^{1}$ is determined later, then we have

$$
\begin{aligned}
u_{1}^{-1} P_{\rho, m}(s, & \left.y ; D_{s}, D_{y}\right)\left(u_{1}\right) \\
= & \sum_{j, \alpha^{\prime}} \rho^{\left.\left(\kappa-\mu_{0}+2\right)\left(j+\left|a^{\prime}\right|\right)+\left(\mu_{0}-1\right) m-\nu\right\} \delta+m-j-\left|a^{\prime}\right|+\sigma_{1}\left(j+\left|\alpha^{\prime}\right|\right)} \\
& \times s^{\nu-\kappa\left(j+\left|\alpha^{\prime}\right|\right)+\left(\mu_{0}-1\right) \mid \alpha^{\prime}} b_{j, a^{\prime}}^{(0)}\left(\rho^{-\delta} s, \rho^{-\mu_{0} \delta} y^{\prime}, \rho^{-\left(\mu_{0}-1\right) \delta} y_{n}\right) \\
& \times\left\{\gamma^{m-j-\left|\alpha^{\prime}\right|}\left(\partial_{s} l^{1}\right)^{j}\left(\partial_{y^{\prime}} l^{1}\right)^{\alpha^{\prime}}+o(1)\right\} \quad(\rho \rightarrow \infty) .
\end{aligned}
$$

Put

and

$$
\begin{aligned}
d_{j, \alpha^{\prime}}^{(0)} & =\left\{\left(\kappa-\mu_{0}+2\right)\left(j+\left|\alpha^{\prime}\right|\right)+\left(\mu_{0}-1\right) m-\nu\right\} \delta+m-j-\left|\alpha^{\prime}\right|+\sigma_{1}\left(j+\left|\alpha^{\prime}\right|\right) \\
& =\left\{\left(\kappa-\mu_{0}+2\right) \sigma_{1}\left(j+\left|\alpha^{\prime}\right|\right)+m(\kappa+1)-\nu\right\} \delta,
\end{aligned}
$$

$$
d_{\max }=\left\{\left(\kappa-\mu_{0}+2\right) \sigma_{1} m^{*}+m(\kappa+1)-\nu\right\} \delta .
$$

Note that when $j+\left|\alpha^{\prime}\right|>m^{*}$, by (4), we have

$$
b_{j, \alpha^{\prime}}^{(0)}\left(\rho^{-\delta} s, \rho^{-\mu_{0} \delta} y^{\prime}, \rho^{-\left(\mu_{0}-1\right) \delta} y_{n}\right)=\rho^{-\left(\kappa^{*}-\kappa\right)\left(m^{*}-j-\left|a^{\prime}\right|\right) \delta} f_{j, \alpha^{\prime}}(s, y ; \rho),
$$


where $f_{j, \alpha^{\prime}}$ is bounded in $C^{\infty}(B)$ when $\rho \rightarrow \infty$.

(a) When $j+\left|\alpha^{\prime}\right|<m^{*}$, we have

$$
d_{\max }-d_{j, \alpha^{\prime}}^{(0)}=\left(\kappa-\mu_{0}+2\right) \sigma_{1}\left(m^{*}-j-\left|\alpha^{\prime}\right|\right) \delta>0 .
$$

(b) When $j+\left|\alpha^{\prime}\right|=m^{*}$, we have

$$
d_{\max }-d_{j, \alpha^{\prime}}^{(0)}=0 .
$$

(c) When $j+\left|\alpha^{\prime}\right|>m^{*}$, by (4.1), we have

$$
\begin{aligned}
& d_{\max }-\left\{d_{j, \alpha^{\prime}}^{(0)}-\left(\kappa^{*}-\kappa\right)\left(m^{*}-j-\left|\alpha^{\prime}\right|\right) \delta\right\} \\
& =\left\{\left(\kappa-\mu_{0}+2\right) \sigma_{1}+\kappa^{*}-\kappa\right\}\left(m^{*}-j-\left|\alpha^{\prime}\right|\right) \delta>0 .
\end{aligned}
$$

Thus, by (4.4), we have

$$
\begin{aligned}
u_{1}^{-1} P_{\rho, m}\left(u_{1}\right)=\rho^{d_{\max }}\left\{\sum_{j+\left|\alpha^{\prime}\right|=m *} s^{\nu-\kappa m *+\left(\mu_{0}-1\right)\left|\alpha^{\prime}\right|}\right. \\
\\
\left.\times b_{j, \alpha^{\prime}}^{(0)}(0,0) \gamma^{m-m *}\left(\partial_{s} l^{1}\right)^{j}\left(\partial_{y^{\prime}} l^{1}\right)^{\alpha^{\prime}}+o(1)\right\} .
\end{aligned}
$$

By the same way, for $h \geqq 1$, we have

and

$$
\begin{aligned}
P_{\rho, m-h}(s, y ; \sigma, \eta)= & \sum_{j, \alpha^{\prime}} \rho^{\left\{\left(\kappa-\mu_{0}+2\right)\left(j+\left|\alpha^{\prime}\right|+h\right)+\left(\mu_{0}-1\right) m-\nu+\varepsilon\left(h, j, \alpha^{\prime}\right)\right\} \delta} \\
& \times s^{\nu-\kappa\left(j+\left|\alpha^{\prime}\right|+h\right)-h+\left(\mu_{0}-1\right)\left|\alpha^{\prime}\right|-\varepsilon\left(h, j, \alpha^{\prime}\right)} b_{j, \alpha^{\prime}}^{(h)} \\
& \times \sigma^{j} \eta^{\prime \alpha^{\prime}} \eta_{\eta n}^{m-h-j-\left|\alpha^{\prime}\right|}
\end{aligned}
$$

$$
\begin{aligned}
u_{1}^{-1} P_{\rho, m-h}\left(u_{1}\right)= & \sum_{j, \alpha^{\prime}} \rho^{\left\{\left(\kappa-\mu_{0}+2\right) \sigma_{1}\left(j+\left|\alpha^{\prime}\right|\right)+m(\kappa+1)-\nu+\varepsilon\left(h, j, \alpha^{\prime}\right)\right\} \delta} \\
& \times s^{\nu-\kappa\left(j+\mid \alpha^{\prime} i+h\right)-h+\left(\mu_{0}-1\right)\left|\alpha^{\prime}\right|-\varepsilon\left(h, j, \alpha^{\prime}\right)} \\
& \times b_{j, \alpha^{\prime}}^{(h)}\left(\rho^{-\delta} s, \rho^{-\mu_{0} \delta} y^{\prime}, \rho^{-\left(\mu_{0}-1\right) \delta} y_{n}\right) \\
& \times\left\{\gamma^{m-h-j-\left|\alpha^{\prime}\right|}\left(\partial_{s} l^{1}\right)^{j}\left(\partial_{y^{\prime}} l^{1}\right)^{\alpha^{\prime}}+o(1)\right\}
\end{aligned}
$$

We put $d_{j, \alpha^{\prime}}^{(h)}=\left\{\left(\kappa-\mu_{0}+2\right) \sigma_{1}\left(j+\left|\alpha^{\prime}\right|\right)+m(\kappa+1)-\nu+\varepsilon\left(h, j, \alpha^{\prime}\right)\right\} \delta$.

(d) When $h+j+\left|\alpha^{\prime}\right|<m^{*}$, we have

$$
d_{\max }-d_{j, \alpha^{\prime}}^{(h)}=\left\{\left(\kappa-\mu_{0}+2\right) \sigma_{1}\left(m^{*}-j-\left|\alpha^{\prime}\right|\right)-\varepsilon\left(h, j, \alpha^{\prime}\right)\right\} \delta \geqq 0 .
$$

The equality holds if and only if $\left(h, j, \alpha^{\prime}\right) \in \mathfrak{M}$.

(e) When $h+j+\left|\alpha^{\prime}\right| \geqq m^{*}$, by (6) and (4.1), we have

$$
\begin{aligned}
& d_{\max }-d_{j, \alpha^{\prime}}^{(h)} \geqq\left\{\left(\kappa-\mu_{0}+2\right) \sigma_{1}\left(m^{*}-j-\left|\alpha^{\prime}\right|\right)+\left(\kappa^{*}-\kappa\right)\left(m^{*}-h-j-\left|\alpha^{\prime}\right|\right)\right\} \delta \\
& =\left[\left(m^{*}-h-j-\left|\alpha^{\prime}\right|\right)\left\{\left(\kappa-\mu_{0}+2\right) \sigma_{1}+\kappa^{*}-\kappa\right\}+h\left(\kappa-\mu_{0}+2\right) \sigma_{1}\right] \delta>0 .
\end{aligned}
$$

Thus, we have

where

$$
u_{1}^{-1} P_{\rho}\left(u_{1}\right)=\rho^{d_{\max }}\left\{\gamma^{m-m *} \Phi_{1}\left(s, \gamma ; \partial_{s} l^{1}, \partial_{y} l^{1}\right)+o(1)\right\} \quad(\rho \rightarrow \infty) .
$$




$$
\begin{aligned}
\Phi_{1}\left(s, \gamma ; \sigma, \eta^{\prime}\right)= & \sum_{j+\left|\alpha^{\prime}\right|=m *} s^{\nu-\kappa m *+\left(\mu_{0}-1\right)\left|\alpha^{\prime}\right|} b_{j, \alpha^{\prime}}^{(0)}(0,0) \sigma^{j} \eta^{\prime \alpha^{\prime}} \\
+ & \sum_{\left(h, j, \alpha^{\prime}\right) \in \mathbb{M}_{0}} s^{\nu-\kappa\left(j+\left|\alpha^{\prime}\right|+h\right)-h+\left(\mu_{0}-1\right)\left|\alpha^{\prime}\right|-\varepsilon\left(h, j, \alpha^{\prime}\right)} \\
& \times b_{j, \alpha^{\prime}}^{(h)}(0,0) \gamma^{m *-h-j-\left|\alpha^{\prime}\right|} \sigma^{j} \eta^{\prime \alpha^{\prime}} .
\end{aligned}
$$

Note that $m^{*}-h-j-\left|\alpha^{\prime}\right|>0$ for $\left(h, j, \alpha^{\prime}\right) \in \mathfrak{M}_{0}$.

By the same way as the proof of Theorem 4.1 in [2] with $m^{*}$ acting as $r$, we have the following.

For sufficiently large $N$, there exist real numbers $\gamma_{0}, \theta, H$, an open set $Y \subset$ $\left\{(s, y) \in \boldsymbol{R}^{n+1} ; s>0\right\}$, positive integers $d, g, K$, functions $l^{j}(s, y) \in C^{\infty}(Y)(j=1$, $\cdots, d)$, rational numbers $\sigma_{1}>\sigma_{2}>\cdots>\sigma_{d}>0$ and differential operators $L_{r}(r=0,1$, $\cdots, N), L_{N+1, \rho}$ such that the followings are satisfied.

1)

$$
\operatorname{Im} \partial_{s} l^{1}(s, v)<-\theta<0 \quad \text { on } Y
$$

2)

3)

$$
\left|\operatorname{grad}_{y} \operatorname{Im} l^{1}(s, y)\right| \leqq \frac{\theta}{4 C_{0} n} \quad \text { on } \quad Y
$$

$$
E_{\rho}^{-1} \circ P_{\rho} \circ E_{\rho}=\rho^{H}\left\{L_{0}+\sum_{r=1}^{N} \rho^{-r / K} L_{r}+\rho^{-(N+1) / K} L_{N+1, \rho}\right\},
$$

where $E_{\rho}(s, y)=\exp \left(i \gamma_{0} y_{n} \rho+i \sum_{j=1}^{d} l^{j}(s, y) \rho^{\sigma_{j}}\right)$.

4) $L_{r}(r=0, \cdots, N)$ have analytic coefficients on $Y$, ord. $L_{r} \leqq m$ and $L_{0}=$ $\sum_{j+|\alpha| \leq g} b_{j, \alpha}(s, y) D_{s}^{j} D_{y}^{\alpha}$, where $b_{g, 0}(s, y) \neq 0$ on $Y$.

5) $L_{N+1, \rho}$ have coefficients which are bounded in $C^{\infty}(Y)$ when $\rho \rightarrow \infty$ and ord. $L_{N+1, \rho} \leqq m$.

We fix $(\hat{s}, \hat{y}) \in Y$. In a sufficiently small neighborhood $Z$ of $(\hat{s}, \hat{y})$, we can solve the following equation:

$$
\left\{\begin{array}{l}
\left(\sum_{r=0}^{N} \rho^{-r / K} L_{r}\right)\left(\sum_{\jmath=0}^{N} \rho^{-j / K} v_{\jmath}(s, y)\right)=O\left(\rho^{-(N+1) / K}\right), \\
v_{0}(\hat{s}, \tilde{y})=1 .
\end{array}\right.
$$

We put $t_{\rho}=\rho^{-\delta} \hat{s}, \quad x_{\rho, j}=\rho^{-\mu_{0} \delta} \hat{y}_{j} \quad(j=1, \cdots, n-1), \quad x_{\rho, n}=\rho^{-\left(\mu_{0}-1\right) \delta} \hat{y}_{n}$ and $\Gamma_{\rho}=$ $\Gamma_{\mu_{0}}\left(t_{\rho}, x_{\rho} ; C_{0}\right)$. Under the coordinate transformation (4.2), $\Gamma_{\rho}$ is transformed into

$$
\begin{aligned}
\Gamma_{\rho}^{*} & =\left\{(s, y) ;\left|y^{\prime}-\hat{y}^{\prime}\right|^{2}+\rho^{2 \delta}\left|y_{n}-\hat{y}_{n}\right|^{2} \leqq C_{0}^{2}\left(\hat{s}^{\mu_{0}}-s^{\mu_{0}}\right)^{2}, 0 \leqq s \leqq \hat{s}\right\} \\
& \subset\left\{(s, y) ;|y-\hat{y}| \leqq C_{1}(\hat{s}-s), 0 \leqq s \leqq \hat{s}\right\} \quad \text { for } \quad \rho \geqq 1 .
\end{aligned}
$$

For $(s, y) \in Y \cap \Gamma_{\rho}^{*}$, we have by (4.7), 


$$
\begin{aligned}
\operatorname{Im} l^{1}(s, y) \geqq & \operatorname{Im} l^{1}(\hat{s}, \hat{y})+\partial_{s} \operatorname{Im} l^{1}(\hat{s}, \hat{y})(s-\hat{s}) \\
& +\sum_{j=1}^{n} \partial_{y_{j}} \operatorname{Im} l^{1}(\hat{s}, \hat{y})\left(y_{j}-\hat{y}_{j}\right)-C_{2}\left(|s-\hat{s}|^{2}+|y-\hat{y}|^{2}\right) \\
\geqq & \operatorname{Im} l^{1}(\hat{s}, \hat{y})+\partial_{s} \operatorname{Im} l^{1}(\hat{s}, \hat{y})(s-\hat{s})-\frac{\theta}{2}|s-\hat{s}| \\
& -C_{2}\left(1+C_{1}^{2}\right)|s-\hat{s}|^{2} .
\end{aligned}
$$

By (4.6), there exist a neighborhood $Z_{1}$ of $(\hat{s}, \hat{y})$ and a positive number $\varepsilon_{0}$ such that

$$
\operatorname{Im} l^{1}(s, y) \geqq \operatorname{Im} l^{1}(\hat{s}, \hat{y})+\varepsilon_{0}(\hat{s}-s) \quad \text { for any } \quad(s, y) \in Z_{1} \cap \Gamma_{\rho}^{*} .
$$

From the assumption of $\left(\mu_{0}\right)$-well-posedness, there exist a positive integer $q$ and positive constants $J, C_{3}$ such that

$$
\|u\|_{0, \rho}^{\prime} \leqq C_{3} \rho^{J}\left\|P_{\rho} u\right\|_{q, \rho}^{\prime} \quad \text { for any } \quad u \in C_{f}^{\infty}\left([0, T] \times \boldsymbol{R}^{n}\right),
$$

where $\|\cdot\|_{q, \rho}^{\prime}$ denotes the $C^{q}$-norm on $\Gamma_{\rho}^{*}$, and $\rho \geqq 1$. We choose $\delta^{\prime}>0$ such that $\Gamma_{\rho}^{*} \cap\left\{(s, y) ; \hat{s}-\delta^{\prime} \leqq s \leqq \hat{s}\right\} \subset Z_{1}$ and put $\omega_{\rho}=\Gamma_{\rho}^{*} \cap\left\{(s, y) ; \hat{s}-\delta^{\prime} \leqq s \leqq \hat{s}-\delta^{\prime} / 2\right\}$. We take $\phi(s) \in C^{\infty}(\boldsymbol{R})$ such that $0 \leqq \psi(s) \leqq 1$ and

We define

$$
\psi(s)= \begin{cases}0, & s \leqq \hat{s}-\delta^{\prime} \\ 1, & s \geqq \hat{s}-\delta^{\prime} / 2 .\end{cases}
$$

$$
u_{\rho}=\phi(s) E_{\rho}(s, y) \sum_{j=0}^{N} v_{j}(s, y) \rho^{-j / K} .
$$

Then, for sufficiently large $N$, there exist positive constants $C_{4}, C_{5}, C_{6}$ such that

and

$$
\left\|P_{\rho} u_{\rho}\right\|_{q, \rho}^{\prime} \leqq C_{4} \rho^{-J-1} \underset{\Gamma_{o}^{*}}{\sup }\left|\psi E_{\rho}\right|+C_{5} \rho^{H+q_{\omega_{\rho}}} \sup _{\rho}\left|E_{\rho}\right|
$$

$$
\left\|u_{\rho}\right\|_{0, \rho}^{\prime} \geqq\left(1 / 2-C_{6} \rho^{-1}\right) \sup _{\Gamma_{\rho}^{*}}\left|\phi E_{\rho}\right|,
$$

for sufficiently large $\rho$. So, by (4.9), there exists a constant $C_{7}$ such that

And we have

$$
\left|E_{\rho}(\hat{s}, \hat{y})\right| \leqq \sup _{\Gamma_{\rho}^{*}}\left|\phi E_{\rho}\right| \leqq C_{7} \rho^{H+q+J} \sup _{\omega_{\rho}}\left|E_{\rho}\right| .
$$

This contradicts (4.8).

$$
\operatorname{Im} l^{1}(\hat{s}, \hat{y})+\sup _{\omega_{\rho}}\left\{-\operatorname{Im} l^{1}(s, y)\right\} \geqq 0 .
$$

\section{$\S 5$. Proof of Theorem 2}

By Proposition 2.2, 2.4, we may assume that $M=1, \mu_{0}, \nu_{r}, \kappa_{r}(r=1, \cdots, l)$ are positive integers, $\mu_{0} \geqq 2$ and $\tau_{0}=0$, without loss of generality. We have only to prove that

$$
b_{j, \alpha^{\prime}}^{(h)}(0,0)=0 \text { for } h+j+\left|\alpha^{\prime}\right|<d,
$$

under the following assumptions :

(1) $\partial_{\tau}^{j} \partial_{\xi^{\prime}}^{\alpha^{\prime}} P_{m-h}\left(t, x ; 0, e_{n}\right)=j ! \alpha^{\prime} ! t^{\nu-\kappa\left(j+\left|\alpha^{\prime}\right|+h\right)-h+\left(\mu_{0}-1\right)\left|\alpha^{\prime}\right|} b_{j, \alpha^{\prime}}^{(h)}(t, x)$, 
where $b_{j, \alpha^{\prime}}^{(h)} \in C^{\infty}\left(\Omega^{+}\right)$and $\nu, \kappa$ are positive integers.

(2) $b_{j, 0}^{(0)}(0,0)=0$ for $j<d$.

(3) $b_{d, 0}^{(0)}(0,0) \neq 0$.

(2) means that there exists a positive number $\varepsilon$ such that

$$
\partial_{\tau}^{j} P_{m}\left(t, 0 ; 0, e_{n}\right) \in t^{\nu+\varepsilon d-(\kappa+\varepsilon) j} \times C^{\infty} .
$$

So, by Proposition 3.2, we have

$$
\begin{aligned}
\partial_{\tau}^{j} \partial_{\xi^{\prime}}^{\alpha^{\prime}} P_{m}\left(t, 0 ; 0, e_{n}\right) & \in t^{\nu+\varepsilon d-(\kappa+\varepsilon)\left(j+\left|\alpha^{\prime}\right|\right)+\left(\mu_{0}-1\right)\left|\alpha^{\prime}\right|} \times C^{\infty} \\
& =t^{\nu-\kappa\left(j+\left|\alpha^{\prime}\right|\right)+\left(\mu_{0}-1\right)\left|\alpha^{\prime}\right|+\varepsilon\left(d-j-\left|\alpha^{\prime}\right|\right)} \times C^{\infty} .
\end{aligned}
$$

This implies (5.1) for $h=0$.

Put $\mathfrak{M}_{1}=\left\{\left(h, j, \alpha^{\prime}\right) ; h+j+\left|\alpha^{\prime}\right|<d, b_{j, \alpha^{\prime}}^{(h)}(0,0) \neq 0\right\}$. We assume that $\mathfrak{M}_{1} \neq \varnothing$. Put

$$
\sigma_{1}=\frac{\varepsilon}{\kappa+\varepsilon-\mu_{0}+2} \max \left\{\frac{d-h-j-\left|\alpha^{\prime}\right|}{d-j-\left|\alpha^{\prime}\right|} ;\left(h, j, \alpha^{\prime}\right) \in \mathfrak{M}_{1}\right\},
$$

where $\varepsilon$ is a positive rational number determined later. Then, we have

$$
0<\sigma_{1}<\frac{\varepsilon}{\kappa+\varepsilon-\mu_{0}+2} .
$$

Put $\mathfrak{M}_{2}=\left\{\left(h, j, \alpha^{\prime}\right) \in \mathfrak{M}_{1} ; \sigma_{1}=\frac{\varepsilon}{\kappa+\varepsilon-\mu_{0}+2} \cdot \frac{d-h-j-\left|\alpha^{\prime}\right|}{d-j-\left|\alpha^{\prime}\right|}\right\}$.

Now, we perform the coordinate transformation (4.2) where $\delta=\left(\kappa+\varepsilon-\mu_{0}+2\right)^{-1}$. As in the proof of Theorem 4.1, $P$ is transformed into $P_{\rho}$ and

$$
\begin{aligned}
u_{1}^{-1} P_{\rho, m-h}\left(u_{1}\right)= & \sum_{j, \alpha^{\prime}} \rho^{d\left(h, j, \alpha^{\prime}\right)} s^{\nu-\kappa\left(j+\left|\alpha^{\prime}\right|+h\right)-h+\left(\mu_{0}-1\right)\left|a^{\prime}\right|} \\
& \times b_{j, \alpha^{\prime}}^{(h)}\left(\rho^{-\delta} s, \rho^{-\mu_{0} \delta} y^{\prime}, \rho^{-\left(\mu_{0}-1\right) \delta} y_{n}\right) \\
& \times\left\{\gamma^{m-j-\left|\alpha^{\prime}\right|-h}\left(\partial_{s} l^{1}\right)^{j}\left(\partial_{y^{\prime}} l^{1}\right)^{a^{\prime}}+o(1)\right\} .
\end{aligned}
$$

where $u_{1}=\exp \left\{i y_{n} \gamma \rho+i l^{1}\left(s, y^{\prime}\right) \rho^{\sigma_{1}}\right\}, d\left(h, j, \alpha^{\prime}\right)=\left[\left\{\left(\kappa+\varepsilon-\mu_{0}+2\right) \sigma_{1}-\varepsilon\right\}\left(j+\left|\alpha^{\prime}\right|\right)+\right.$ $(\kappa+\varepsilon+1) m-\nu-\varepsilon h] \delta$ and $h=0,1, \cdots, m$. Put $d_{\max }=\left[\left\{\left(\kappa+\varepsilon-\mu_{0}+2\right) \sigma_{1}-\varepsilon\right\} d+\right.$ $(\kappa+\varepsilon+1) m-\nu] \delta$.

Note that $b_{j, \alpha^{\prime}}^{(h)}\left(\rho^{-\delta} s, \rho^{-\mu_{0} \delta} y^{\prime}, \rho^{-\left(\mu_{0}-1\right) \delta} y_{n}\right)=O\left(\rho^{-\delta}\right)$ if $b_{j, a^{\prime}}^{(h)}(0,0)=0$, especially if $h=0, j+\left|\alpha^{\prime}\right|<d$.

(a) For sufficiently small $\varepsilon$, we have

$$
d_{\max }-\left\{d\left(h, j, \alpha^{\prime}\right)-\delta\right\}=\left[\left\{\left(\kappa+\varepsilon-\mu_{0}+2\right) \sigma_{1}-\varepsilon\right\}\left(d-j-\left|\alpha^{\prime}\right|\right)+\varepsilon h+1\right] \delta>0 .
$$

(b) When $h=0, j+\left|\alpha^{\prime}\right|=d$, we have

$$
d_{\max }-d\left(0, j, \alpha^{\prime}\right)=0 .
$$

(c) When $h=0, j+\left|\alpha^{\prime}\right|>d$ or $h \geqq 1, h+j+\left|\alpha^{\prime}\right| \geqq d$, we have

$$
\begin{gathered}
d_{\max }-d\left(h, j, \alpha^{\prime}\right)=\left\{\left(\kappa+\varepsilon-\mu_{0}+2\right) \sigma_{1}-\varepsilon\right\}\left(d-h-j-\left|\alpha^{\prime}\right|\right) \\
+\left(\kappa+\varepsilon-\mu_{0}+2\right) \sigma_{1} h>0 .
\end{gathered}
$$

(d) When $\left(h, j, \alpha^{\prime}\right) \in \mathfrak{M}_{1}$, we have 


$$
d_{\max }-d\left(h, j, \alpha^{\prime}\right)=\left\{\left(\kappa+\varepsilon-\mu_{0}+2\right) \sigma_{1}\left(d-j-\left|\alpha^{\prime}\right|\right)-\varepsilon\left(d-h-j-\left|\alpha^{\prime}\right|\right)\right\} \delta \geqq 0 .
$$

The equality holds for $\left(h, j, \alpha^{\prime}\right) \in \mathfrak{M}_{2}$.

Thus, if we take $\varepsilon$ sufficiently small, then we have

where

$$
u_{1}^{-1} P_{\rho}\left(u_{1}\right)=\rho^{d_{\max }}\left\{\gamma^{m-d} \Phi_{1}\left(s, \gamma ; \partial_{s} l^{1}, \partial_{y^{\prime}} l^{1}\right)+o(1)\right\},
$$

$$
\begin{aligned}
\Phi_{1}\left(s, \gamma ; \sigma, \eta^{\prime}\right)= & \sum_{j+\left|\alpha^{\prime}\right|=d} s^{\nu-\kappa d+\left(\mu_{0}-1\right) \mid \alpha^{\prime} !} b_{j, \alpha^{\prime}}^{(0)}(0,0) \sigma^{j} \eta^{\prime \alpha^{\prime}} \\
+ & \sum_{\left(h, j, \alpha^{\prime}\right) \in \mathfrak{M}_{2}} s^{\nu-\kappa\left(j+\left|\alpha^{\prime}\right|+h\right)-h+\left(\mu_{0}-1\right) ; \alpha^{\prime} \mid} b_{j, \alpha^{\prime}}^{(h)}(0,0) \\
& \times \gamma^{d-h-j-\left|\alpha^{\prime}\right|} \sigma^{j} \eta^{\prime \alpha^{\prime}} .
\end{aligned}
$$

The rest of the proof is just the same as the proof of Proposition 4.1, with $d$ acting as $m^{*}$.

\section{$\S 6$. Proof of Theorem 3}

By Proposition 2.4, we may assume that $\tau_{0}=0$, without loss of generality. So, we assume

(1) $B_{j, \alpha^{\prime}}^{(h)}=b_{j, \alpha^{\prime}}^{(h)}(0,0)$,

(2) $\partial_{\tau}^{j} \partial_{\xi^{\prime}}^{\alpha^{\prime}} P_{m-h}\left(t, x ; 0, e_{n}\right)=j ! \alpha^{\prime} ! t^{\nu-\kappa\left(j+\left|\alpha^{\prime}\right|+h\right)-h+\left(\mu_{0}-1\right)\left|\alpha^{\prime}\right|} b_{j, \alpha^{\prime}}^{(h)}(t, x)$,

where $b_{j, \alpha^{\prime}}^{(h)} \in E_{M}\left(\Omega^{+}\right)$,

(3) $b_{j, 0}^{(h)}(t, 0)=t^{\nu_{0}(d-j-h)} g_{j}^{(h)}(t)$ for $j+h \leqq d-1$,

where $g_{j}^{(h)} \in C^{0}[0, T], \nu_{0}$ is a positive rational number,

(4) $\quad b_{d, 0}^{(0)}(0,0) \neq 0$.

We perform the following coordinate transformation:

$$
\left\{\begin{array}{l}
t=\rho^{-\omega_{0}} s, \\
x_{j}=\rho^{-\omega^{\prime}} y_{j} \\
x_{n}=\rho^{-\omega_{n}} y_{n},
\end{array} \quad(j=1, \cdots, n-1),\right.
$$

where $\omega_{0}, \omega^{\prime}, \omega_{n}$ are positive integers determined later which depend only on $M, \mu_{0}, m, \kappa, \nu . P$ is transformed into $P_{\rho}$ which is a differential operator on $B$ for sufficiently large $\rho$, and

$$
\begin{aligned}
P_{\rho, m-h}(s, y ; \sigma, \eta)= & P_{m-h}\left(s \rho^{-\omega_{0}}, y \rho^{-\omega} ; \sigma \rho^{\omega_{0}}, \eta \rho^{\omega}\right) \\
= & \eta_{n}^{m-h} \rho^{\omega_{n}(m-h)} \sum_{j+\left|\alpha^{\prime}\right| \leq m-h} \rho^{j\left(\omega_{0}-\omega_{n}\right)+\left|\alpha^{\prime}\right|\left(\omega^{\prime}-\omega_{n}\right)} \\
& \quad \times \partial_{\tau}^{j} \partial_{\xi^{\prime}}^{\alpha^{\prime}} P_{m-h}\left(s \rho^{-\omega_{0}}, y \rho^{-\omega} ; 0, e_{n}\right) \frac{1}{j ! \alpha^{\prime} !}\left(\frac{\sigma}{\eta n}\right)^{j}\left(\frac{\eta^{\prime}}{\eta_{n}}\right)^{\alpha^{\prime}} \\
= & \sum_{j+\left|\alpha^{\prime}\right| \leq m-h} \rho^{d\left(h, j, \alpha^{\prime}\right)}\left\{s^{\nu-\kappa\left(j+\left|\alpha^{\prime}\right|+h\right)-h+\left(\mu_{0}-1\right)\left|\alpha^{\prime}\right|}\right. \\
& \left.\quad \times b_{j, \alpha^{\prime}}^{(h)}\left(s \rho^{-\omega_{0}}, 0\right) \sigma^{j} \eta^{\prime \alpha^{\prime}} \eta_{n}^{m-h-j-\left|\alpha^{\prime}\right|}+o(1)\right\},
\end{aligned}
$$

where $d\left(h, j, \alpha^{\prime}\right)=\left\{(\kappa+1)\left(h+j+\left|\alpha^{\prime}\right|\right)-\mu_{0}\left|\alpha^{\prime}\right|-\nu\right\} \omega_{0}+\left|\alpha^{\prime}\right| \omega^{\prime}+\left(m-h-j-\left|\alpha^{\prime}\right|\right) \omega_{n}$. Put $d_{\max }=\{(\kappa+1) d-\nu\} \omega_{0}+(m-d) \omega_{n}$. 
We want to take $\omega_{0}, \omega^{\prime}, \omega_{n}$ such that

(a) $d_{\max }>d(h, j, 0)$ when $j+h>d$,

(b) $d_{\max }>d(h, j, 0)-\nu_{0}(d-j-h) \omega_{0}$ when $j+h<d$,

(c) $d_{\max }>d\left(h, j, \alpha^{\prime}\right)$ when $\left|\alpha^{\prime}\right|>0$.

If these are satisfied, then we have

$$
P_{\rho}(s, y ; \sigma, \eta)=\rho^{d} \max \left\{\sum_{j+h=d} s^{\nu-\kappa d-h} b_{j, 0}^{(h)}(0,0) \sigma^{j} \eta_{n}^{m-d}+o(1)\right\} .
$$

Now, (a) means that

$$
\omega_{n}>(\kappa+1) \omega_{0},
$$

and (b) means that

$$
\left\{(\kappa+1)+\nu_{0}\right\} \omega_{0}>\omega_{n} .
$$

We take $\omega_{n}=(\kappa+1+\varepsilon) \omega_{0}$, where $0<\varepsilon<\nu_{0}$. Then, (c) means that

$$
\left\{\left(h+j+\left|\alpha^{\prime}\right|-d\right) \varepsilon+\mu_{0}\left|\alpha^{\prime}\right|\right\} \omega_{0}>\left|\alpha^{\prime}\right| \omega^{\prime} \quad \text { if } \quad\left|\alpha^{\prime}\right|>0 .
$$

If we take $\varepsilon<\frac{\nu_{0}}{2 d}$ and $\omega^{\prime}<\frac{1}{2} \mu_{0} \omega_{0}$, then (6.5) holds.

Thus, we can take $\omega_{0}, \omega^{\prime}, \omega_{n}, \varepsilon$ depending only on $M, \mu_{0}, m, \nu, \kappa$ such that

$$
\begin{aligned}
P_{\rho}\left(s, y ; D_{s}, D_{y}\right)=\rho^{a_{\max }}\left\{\sum_{j+h=d} s^{\nu-\kappa d-h} b_{j, 0}^{(h)}(0,0) D_{s}^{j} D_{y_{n}}^{m-d}\right. \\
\left.\quad+\sum_{j=1}^{N} \rho^{-j} R_{\jmath}\left(s, y ; D_{s}, D_{y}\right)+\rho^{-(N+1)} R_{N+1, \rho}\left(s, y ; D_{s}, D_{y}\right)\right\} .
\end{aligned}
$$

Here, $N$ is a sufficiently large integer, $R_{j} \in Q(m)=\left\{R\left(s, y ; D_{s}, D_{y}\right)\right.$; a differential operator on $B$, ord. $R \leqq m$, and $R$ satisfies $\left(A-M, \mu_{0}\right)$ on $\left.B\right\}(j=1, \cdots, N)$ and $R_{N+1, \rho} \in Q^{*}(m)=\left\{R_{\rho}\left(s, y ; D_{s}, D_{y}\right)\right.$; a differential operator on $B$, ord. $R_{\rho} \leqq m$, and the coefficient of $D_{s}^{j} D_{y}^{\alpha}$ is bounded in $t^{j+\mu_{0}\left|\alpha^{\prime}\right|-m} \times E_{M}(B)$ when $\left.\rho \rightarrow \infty\right\}$. Further, $R_{j}(j=1, \cdots, d)$ do not contain the terms $D_{s}^{k} D_{y}^{\alpha}$ where $\alpha_{n} \geqq m-d+1$. Put

$$
L=A^{-1} \sum_{h=0}^{d} b_{d-h, 0}^{(h)}(0,0) s^{d-h} D_{s}^{d-h}, \quad A=i^{-d} b_{d, 0}^{(0)}(0,0) .
$$

Then, by the definition of $\lambda_{l}(l=1, \cdots, d)$, we have

$$
L=\prod_{l=1}^{d}\left(s \partial_{s}-i \lambda_{l}\right)
$$

$$
P_{\rho}=\rho^{d} \max _{\alpha} A\left\{s^{\nu-\kappa d-d} L D_{y_{n}}^{m-d}+\sum_{j=1}^{N} \rho^{-j} R_{\jmath}+\rho^{-(N+1)} R_{N+1} \rho\right\} .
$$

Now,

$$
\begin{aligned}
& \exp \left(-i \rho y_{n}\right) \circ P_{\rho^{\circ}} \exp \left(i \rho y_{n}\right) \\
& \quad=\rho^{d \max ^{+m-d}} A\left\{s^{\nu-\kappa d-d} L+\sum_{j=1}^{N} \rho^{-j} S_{\jmath}+\rho^{-(N+1)} S_{N+1} \rho\right\},
\end{aligned}
$$

where $S_{j} \in \mathbb{Q}(m)(j=1, \cdots, N), S_{N+1, \rho} \in Q^{*}(m)$. We may assume that Re $i \lambda_{1}=$ $\max _{1 \leqq l \leqq d} \operatorname{Re} i \lambda_{l}$. Set $\theta=i \lambda_{1}$, then $-\operatorname{Im} \lambda_{1}=\operatorname{Re} \theta$. We may assume that $\operatorname{Re} \theta \geqq 0$. By 
the fact $\left(s \partial_{s}-i \lambda_{l}\right) \circ s^{\theta}=s^{\theta} \circ\left(s \partial_{s}-i \lambda_{l}+\theta\right)$, we have

$$
\left\{\begin{array}{l}
s^{-\theta} \circ \exp \left(-i \rho y_{n}\right) \circ P_{\rho} \circ \exp \left(i \rho y_{n}\right) \circ s^{\theta}=\rho^{d_{\max }+m-d} A L_{\rho}, \\
L_{\rho}=s^{\nu-\kappa d-d} L_{0}+\sum_{j=1}^{N} \rho^{-j} T_{j}+\rho^{-(N+1)} T_{N+1, \rho},
\end{array}\right.
$$

where $L_{0}=s^{-\theta} \circ L \circ s^{\theta}=\prod_{l=1}^{d}\left(s \partial_{s}+i \mu_{l}\right), \mu_{l}=\lambda_{1}-\lambda_{l}$ and $T_{j} \in \mathbb{Q}(m)(j=1, \cdots, N), T_{N+1, \rho}$ $\in Q^{*}(m)$. Note that $\operatorname{Re} i \mu_{l} \geqq 0(l=1, \cdots, d)$. Define

$$
E_{c}(B)=\left\{f \in E_{M}\left([0,1] \times \mathbb{R}^{n}\right) ; \operatorname{supp} f \subset B\right\} .
$$

Then, we have the following lemma.

Lemma 6.1. For complex numbers $\mu, \alpha$, a non-negative integer $j$ and $f \in E_{c}(B)$, we consider

$$
\left(s \partial_{s}+i \mu\right) v=s^{\alpha}(\log s)^{j} f(s, y), \quad s \in[0,1] .
$$

There exist $g_{h} \in E_{c}(B)$ and $A_{h} \in C_{0}^{\infty}\left(B^{\prime}\right) \quad(h=0, \cdots, j)$ such that the following function is a solution of (6.10).

$$
v(s, y)=s^{\alpha} \sum_{h=0}^{j} g_{h}(s, y)(\log s)^{j-h}+s^{-i \mu} \sum_{h=0}^{j} A_{h}(y)(\log s)^{j+1-h} \text { 。 }
$$

Further, if $\operatorname{Re} \alpha>-\operatorname{Re} i \mu$, then we can take $A_{h} \equiv 0(h=0, \cdots, j)$.

Proof. For any $G \in C_{0}^{\infty}\left(B^{\prime}\right)$, the function

$$
v(s, y)=s^{-i \mu}\left[\int_{1}^{s} t^{\alpha+i \mu-1}(\log t)^{j} f(t, y) d t+G(y)\right]
$$

is a solution of (6.10). We claim that this has the form of (6.11) for some $G$. Note that we can expand $f$ in the following form;

$$
f(t, y)=\sum_{j=0}^{K} f_{j}(y) t^{j / M}+t^{(K+1) / M} f_{K}^{*}(t, y),
$$

where $f_{\jmath} \in C_{0}^{\infty}\left(B^{\prime}\right)$ and $f_{K}^{*} \in E_{c}(B)$. So, we have

$$
\int_{1}^{s} t^{\alpha+i \mu-1} f(t, y) d t=s^{\alpha+i \mu} g(s, y)+F(y) \log s-G(y),
$$

where $g \in E_{c}(B)$ and $F, G \in C_{0}^{\infty}\left(B^{\prime}\right)$. Further, $F \equiv 0$ if $\operatorname{Re}(\alpha+i \mu)>0$. So, the claim is true for $j=0$.

We assume that the claim is true for $j-1(j \geqq 1)$. Then, by (6.14), we have

$$
\begin{aligned}
& \int_{1}^{s} t^{\alpha+i \mu-1}(\log t)^{j} f(t, y) d t \\
= & {\left[\left\{t^{\alpha+i \mu} g(t, y)+F(y) \log t\right\}(\log t)^{j}\right]_{1}^{s} } \\
& -\int_{1}^{s}\left\{t^{\alpha+i \mu} g(t, y)+F(y) \log t\right\} j(\log t)^{j-1} t^{-1} d t \\
= & s^{\alpha+i \mu} g(s, y)(\log s)^{j}+F(y)(\log s)^{j+1} \\
& -j \int_{1}^{s} t^{\alpha+i \mu-1} g(t, y)(\log t)^{j-1} d t-\frac{j}{j+1} F(y)(\log s)^{j+1} .
\end{aligned}
$$


By induction hypothesis, the claim is true for $j$.

Corollary 6.2. For complex numbers $\mu_{1}, \cdots, \mu_{d}, \alpha$, a non-negative integer $j$ and $f \in E_{c}(B)$, we consider the equation

$$
L_{0} v\left(=\prod_{l=1}^{d}\left(s \partial_{s}+i \mu_{l}\right) v\right)=s^{\alpha}(\log s)^{j} f(s, y) .
$$

There exist $g_{h}, A_{l, h} \in E_{c}(B)(h=0, \cdots, j+d-1 ; l=1, \cdots, d)$ such that the following function is a solution of (6.15).

$$
\begin{aligned}
v(s, y)= & s^{\alpha} \sum_{h=0}^{j} g_{h}(s, y)(\log s)^{j-h} \\
& +\sum_{l \in g(-\operatorname{Re} a)} s^{-i \mu} \sum_{h=0}^{j+d} A_{l, h}(s, y)(\log s)^{j+d-h},
\end{aligned}
$$

where $g(a)=\left\{l ; \operatorname{Re} i \mu_{l} \leqq a\right\}$.

Now, we will solve

$$
L_{\rho}\left(\sum_{j=0}^{N} u_{\jmath} \rho^{-j}\right)=O\left(\rho^{-N-1}\right) .
$$

Take $u_{0}(s, y)=u_{0}(y) \in C_{0}^{\infty}\left(B^{\prime}\right)$, where $u_{0}(0)=1$. Then, $T_{1}\left(u_{0}\right) \in s^{-m} \times E_{c}(B)$. So, by Corollary 6.2 , the equation $s^{\nu-\kappa d-d} L_{0} u_{1}=-T_{1}\left(u_{0}\right)$ has a solution

$$
u_{1}(s, y) \in s^{-m-\nu+(\kappa+1) d} \times E_{c}(B)+\sum_{l \in g(m+\nu-(\kappa+1) d)} s^{-i \mu_{l}} \sum_{h=0}^{d}(\log s)^{d-h} \times E_{c}(B) .
$$

Set $\Delta=m+\nu-(\kappa+1) d+1$. Then, by $\nu-\kappa d \geqq d-m$, we have $\Delta \geqq 1$. Define

$$
\boldsymbol{A}_{j}=\left\{\sum_{l=1}^{j(d+1)} s^{-\alpha} l \sum_{h=0}^{j d}(\log s)^{j d-h} f_{l, h}(s, y) ; \alpha_{l} \in \mathbb{C}, 0 \leqq \operatorname{Re} \alpha_{l} \leqq j \Delta, f_{l, h} \in E_{c}(B)\right\} .
$$

If $u_{j} \in A_{j}(j=1, \cdots, k)$, then there holds $\sum_{l=1}^{k+1} T_{l}\left(u_{k+1-l}\right) \in s^{-m} \times \mathbb{A}_{k}$. So, by Corollary 6.2, the equation $s^{\nu-\kappa d-d} L_{0} u_{k+1}=-\sum_{l=1}^{k+1} T_{l}\left(u_{k+1-l}\right)$ has a solution $u_{k+1} \in \mathbb{A}_{k+1}$. Thus, we get a solution of (6.17) such that $u_{j} \in \mathbb{A}_{j}$.

Take a non-decreasing $C^{\infty}$-function $\chi(t)$ such that

$$
\chi(t)= \begin{cases}0 & (t \leqq 1 / 2), \\ 1 & (t \geqq 1),\end{cases}
$$

and put $\chi_{\rho}(s)=\chi\left(s \rho^{1 /(2 \Delta)}\right)(\rho \geqq 1)$. Note that for $\nu \geqq 1$ there exists $\chi_{\nu} \in C^{\infty}(R)$ such that $\partial_{s}^{\nu} \chi_{\rho}(s)=s^{-\nu} \chi_{\nu}\left(s \rho^{1 /(2 \Delta)}\right)$ and $\operatorname{supp} \chi_{\nu} \subset[1 / 2,1]$. Set

Then,

$$
\left\{\begin{array}{l}
V_{\rho}^{(N)}(s, y)=\sum_{j=0}^{N} u_{j}(s, y) \rho^{-j}, \\
U_{\rho}^{(N)}(s, y)=\exp \left(i \rho y_{n}\right) s^{\theta} \chi_{\rho}(s) V_{\rho}^{(N)} .
\end{array}\right.
$$

$$
\begin{aligned}
P_{\rho}\left(U_{\rho}^{(N)}\right) & =\exp \left(i \rho y_{n}\right) s^{\theta} \rho^{d_{\max ^{+m-d}} A L_{\rho}\left(\chi_{\rho} V_{\rho}^{(N)}\right)} \\
& =\exp \left(i \rho y_{n}\right) s^{\theta-m} \rho^{d_{\max }+m-d}
\end{aligned}
$$


where

$$
\times\left\{\sum_{j=0}^{N} G_{j}^{(N)}(s, y ; \rho) \rho^{-j}+G_{N+1, *}^{(N)}(s, y ; \rho) \rho^{-N-1}\right\},
$$

$$
G_{j}^{(N)} \in \mathbb{B}_{J}=\left\{\sum_{r ; \text { finite }} h_{r}(s, y) \chi_{r}\left(s \rho^{1 /(2 \Delta)}\right) ; h_{r} \in A_{j}, \operatorname{supp} \chi_{r} \subset[1 / 2,1]\right\} \quad(j=0, \cdots, N)
$$

and

$$
\begin{aligned}
G_{N+1, *}^{(N)} \in \boldsymbol{B}_{N+1}^{(*)}=\{ & f(s, y ; \rho)=\sum_{l=1}^{(N+1)(d+1)} \sum_{h=0}^{(N+1) d} \sum_{r ; \text { finite }} s^{-a} l(\log s)^{(N+1) d-h} g_{l, h, r}(s, y ; \rho) \\
& \times \chi_{r}\left(s \rho^{1 /(2 \Delta)}\right) ; 0 \leqq \operatorname{Re} \alpha_{l} \leqq(N+1) \Delta, g_{l, h, r} \text { is bounded in } E_{c}(B) \\
& \text { when } \left.\rho \rightarrow \infty, \operatorname{supp} \chi_{r} \subset[1 / 2,1] \text { or } \chi_{r}=\chi\right\} .
\end{aligned}
$$

The following lemma follows from Lemma 3.10 in [3].

Lemma 6.3. If (1.10) holds for $P$, then there exists a constant $C^{*}$ such that the following inequality holds for sufficiently large $\rho$.

$$
\|u\|_{p}^{(1)} \leqq C^{*} \rho^{(q-p) \omega_{n}}\left\|P_{\rho} u\right\|_{q}^{(2)} \quad \text { for any } \quad u \in C_{0}^{\infty}(B) .
$$

Here,

$$
\begin{aligned}
& \|v\|_{p}^{(1)}= \begin{cases}\left\|D_{y_{n}}^{p} v\right\|_{0} & (p \geqq 0) \\
\|v\|_{p} & (p \leqq 0),\end{cases} \\
& \|v\|_{q}^{(2)}= \begin{cases}\|v\|_{q} & (q \geqq 0) \\
\sup _{w \in C_{0}^{\infty}\left(B^{*}\right)} \frac{|(v, w)|}{\left\|D_{y_{n}}^{q} w\right\|_{0}} & (q \leqq 0),\end{cases}
\end{aligned}
$$

where $\|\cdot\|_{p}$ denotes the $H^{p}$-norm on $B^{*}=[0,1 / 2] \times B^{\prime}$ and $(\cdot, \cdot)$ denotes the $L^{2}$ inner product on $B^{*}$.

Now, the desired result follows from the following lemma, which is proved in the same way as Lemma 3.11 (case (i)) in [3] (note that $\Delta=2(k+1)(n-1), d=1$ and $d_{\max }=4 m-1$ in [3]).

Lemma 6.4. There exist positive constants $\delta, C$ such that

(i) $\left\|U_{\rho}^{(N)}\right\|_{p}^{(1)} \geqq \delta \rho^{p}$,

(ii) $\left\|P_{\rho} U_{\rho}^{(N)}\right\|_{q}^{(2)} \leqq C \rho^{d} \max ^{+m-d+q+(m-\operatorname{Re} \theta) /(2 \Delta)}$

for sufficiently large $\rho$.

From the above two lemmas, we have

$$
p \leqq(q-p) \omega_{n}+d_{\max }+m-d+q+\frac{m-\operatorname{Re} \theta}{2 \Delta} .
$$

So,

$$
\frac{\operatorname{Re} \theta}{2 \Delta} \leqq(q-p)\left(\omega_{n}+1\right)+d_{\max }+m-d+\frac{m}{2 \Delta} .
$$

Since $q-p+-m \geqq 1$, we have

$$
\operatorname{Re} \theta \leqq C(q-p+m),
$$


where $C$ depends only on $M, \mu_{0}, m, \nu, \kappa$.

\section{Appendix. Proof of Remark 4.1}

For any $\hat{x} \in \mathbb{R}^{n}$, we can consider $\hat{x}$ as the origin and define the Newton polygon $\Re_{\hat{x}}$ from $P$. For this $\Re_{\hat{x}}$, we get a function $\mu(j)$, integers $m=j_{0}>j_{1}>$ $\cdots>j_{l}=\hat{j} \geqq 0$ and real numbers $\kappa_{1}^{*} \leqq \cdots \leqq \kappa_{m-\hat{\jmath}}^{*}$ as in Section 1 . We also denote $\kappa_{r}^{*}$ $=\kappa_{r}^{*}(x)$. There exist $g_{r} \in E\left(V^{+}\right)$such that

$$
\partial_{r}^{j_{r}} P_{m}\left(t, x ; 0, e_{n}\right)=t^{\mu\left(j_{r}\right)} g_{r}(t, x) \quad(r=1, \cdots, l),
$$

where $V$ is a neighborhood of $(0, \hat{x})$ and $g_{r}(0, x) \neq 0$ in any neighborhood of $\hat{x}$. We consider the following condition:

$$
g_{r}(0, \hat{x}) \neq 0 \quad(r=1, \cdots, l) .
$$

Note that $\left\{x \in \mathbb{R}^{n} ;\left(B_{\hat{x}}\right)\right.$ holds $\}$ is a dense set. For a Newton polygon $\Omega=$ $\Re\left\{\hat{j} ; \kappa_{1}^{*}, \cdots, \kappa_{m-\hat{\jmath}}^{*}\right\}$ and $\kappa>\kappa_{m-\hat{\jmath}}^{*}$. Put

$$
\Re[\kappa]=\Re\left\{0 ; \kappa_{1}^{*}, \cdots, \kappa_{m-\hat{\jmath}}^{*}, \kappa, \cdots, \kappa\right\} \quad(\kappa \text { appears } \hat{j} \text { times }) .
$$

Then, it is clear from the definition of $\Omega_{\hat{x}}$ that $\Omega_{\hat{x}}$ is continuous with respect to $\hat{x}$ in the following sense: that

For any $\hat{x} \in \mathbb{R}^{n}$ and any $\kappa>\kappa_{m-\hat{\jmath}}^{*}(\hat{x})$, there exists a neighborhood $U$ of $\hat{x}$ such

$$
\Re_{\hat{y}} \subset \Re_{\hat{x}}[\kappa] \quad \text { for any } \hat{y} \in U \text {. }
$$

Now, we assume that Theorem 1 holds under the condition $\left(B_{0}\right)$ and consider the Newton polygon $\Omega=\Re_{0}$. For any $\kappa>\kappa_{m-\hat{\jmath}}^{*}(0)$, we take a neighborhood $U$ of the origin such that (A.1) holds for $\hat{x}=0$. For any $\hat{x} \in U$ and any neighborhood $V$ of $\hat{x}$, we take $\hat{y} \in V$ such that $\left(B_{\hat{y}}\right)$ holds. Then, Theorem 1 holds at $x=\hat{y}$ instead of the origin. Since $V$ is arbitrary and $\eta_{\hat{y}} \subset \Re[\kappa]$, we have

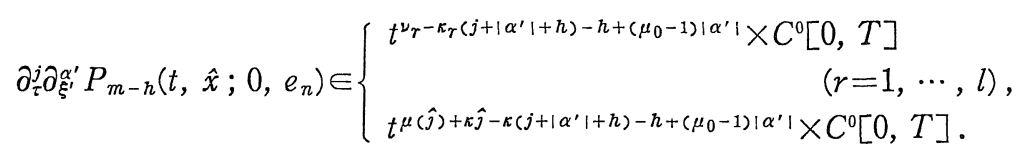

Since $\hat{x}$ and $\kappa$ are arbitrary, we have (1.5).

\section{References}

[1] Flaschka, H. and Strang, G., The correctness of the Cauchy problem, Advances in Math., 6 (1971), 347-379.

[2] Ivrii, V.Y. and Petkov., V.M., Necessary conditions for the Cauchy problem for non-strictly hyperbolic equations to be well-posed, Russian Math. Surveys, 29 (1974), 1-70.

[3] Mandai, T., On energy inequalities and regularity of solutions to weakly hyper- 
bolic Cauchy problems, Publ. RIMS, Kyoto Univ., 18 (1982), to appear.

[4] Sakamoto, R., Cauchy problem for degenerate hyperbolic equations, Comm. Pure Appl. Math., 33 (1980), 785-816.

[5] Tahara, H., Singular hyperbolic systems, III. On the Cauchy problem for Fuchsian hyperbolic partial differential equations, J. Fac. Sci. Univ. Tokyo, 27 (1980), 465507. 